\title{
A Discrete Glycinergic Neuronal Population in the Ventromedial Medulla That Induces Muscle Atonia during REM Sleep and Cataplexy in Mice
}

\author{
Shuntaro Uchida, ${ }^{1,2}{ }^{\circledR}$ Shingo Soya, ${ }^{1,2}$ Yuki C. Saito, ${ }^{2}$ Arisa Hirano, ${ }^{1,2}{ }^{\circledR}$ Keisuke Koga, ${ }^{5}{ }^{\circledR}$ Makoto Tsuda, ${ }^{5}$ \\ ${ }^{(1)}$ Manabu Abe, ${ }^{3}$ Kenji Sakimura, ${ }^{3}$ and ${ }^{(1)}$ Takeshi Sakurai ${ }^{1,2,4}$ \\ ${ }^{1}$ Faculty of Medicine, University of Tsukuba, Tsukuba, Ibaraki 305-8575, Japan, ${ }^{2}$ International Institute for Integrative Sleep Medicine (WPI-IIIS), \\ University of Tsukuba, Tsukuba, Ibaraki 305-8575, Japan, ${ }^{3}$ Department of Cellular Neurobiology, Brain Research Institute, Niigata University, \\ Asahimachi, Chuoku Niigata 951-8585, Japan, ${ }^{4}$ Japan Life Science Center for Tsukuba Advanced Research Alliance (TARA), University of Tsukuba, \\ Tsukuba, Ibaraki 305-8575, Japan, and ${ }^{5}$ Department of Life Innovation, Graduate School of Pharmaceutical Sciences, Kyushu University, Fukuoka \\ 812-8582, Japan
}

During rapid eye movement (REM) sleep, anti-gravity muscle tone and bodily movements are mostly absent, because somatic motoneurons are inhibited by descending inhibitory pathways. Recent studies showed that glycine/GABA neurons in the ventromedial medulla (VMM; Gly ${ }^{\mathrm{VMM}}$ neurons) play an important role in generating muscle atonia during REM sleep (REM-atonia). However, how these REM-atonia-inducing neurons interconnect with other neuronal populations has been unknown. In the present study, we first identified a specific subpopulation of Gly ${ }^{\mathrm{VMM}}$ neurons that play an important role in induction of REM-atonia by virus vector-mediated tracing in male mice in which glycinergic neurons expressed Cre recombinase. We found these neurons receive direct synaptic input from neurons in several brain stem regions, including glutamatergic neurons in the sublaterodorsal tegmental nucleus (SLD; Glu ${ }^{\text {SLD }}$ neurons). Silencing this circuit by specifically expressing tetanus toxin light chain (TeTNLC) resulted in REM sleep without atonia. This manipulation also caused a marked decrease in time spent in cataplexy-like episodes (CLEs) when applied to narcoleptic orexin-ataxin-3 mice. We also showed that Gly ${ }^{\mathrm{VMM}}$ neurons play an important role in maintenance of sleep. This present study identified a population of glycinergic neurons in the VMM that are commonly involved in REM-atonia and cataplexy.

Key words: atonia; cataplexy; glycine; narcolepsy; neural circuit; REM sleep

Significance Statement

We identified a population of glycinergic neurons in the ventral medulla that plays an important role in inducing muscle atonia during rapid eye movement (REM) sleep. It sends axonal projections almost exclusively to motoneurons in the spinal cord and brain stem except to those that innervate extraocular muscles, while other glycinergic neurons in the same region also send projections to other regions including monoaminergic nuclei. Furthermore, these neurons receive direct inputs from several brainstem regions including glutamatergic neurons in the sublaterodorsal tegmental nucleus (SLD). Genetic silencing of this pathway resulted in REM sleep without atonia and a decrease of cataplexy when applied to narcoleptic mice. This work identified a neural population involved in generating muscle atonia during REM sleep and cataplexy.

Received Mar. 24, 2020; revised Dec. 13, 2020; accepted Dec. 15, 2020.

Author contributions: S.U. and T.S. designed research; S.U., S.S., and T.S. performed research; S.S., Y.C.S., A.H., K.K., M.T., M.A., K.S., and T.S. contributed unpublished reagents/analytic tools; S.U., S.S., and T.S. analyzed data; T.S. wrote the paper.

This work was supported by the JSPS KAKENHI Grant-in-Aid for JSPS Fellows $18 J 21114$ (to S.U.), JSPS KAKENHI Grants-in-Aid for Scientific Research (B) JP $15 \mathrm{H} 03122$ and $18 \mathrm{H} 02595$ (to T.S.), the KAKENHI Grant-inAid for Exploratory Research JP 15K12768 (to T.S.), and the KAKENHI Grant-in-Aid for Scientific Research on Innovative Areas "Willdynamics" $16 \mathrm{H} 06401$ (to T.S.). We thank Dr. Kazunari Miyamichi and Mr. Masatoshi Kasuya for technical advice.

The authors declare no competing financial interests.

Correspondence should be addressed to Takeshi Sakurai at sakurai.takeshi.gf@u.tsukuba.ac.jp.

https://doi.org/10.1523/JNEUROSCI.0688-20.2020

Copyright $\odot 2021$ the authors

\section{Introduction}

During rapid eye movement (REM) sleep, all anti-gravity muscle tone and body movements are mostly absent despite the primary motor cortex being activated (REM-atonia; Jouvet, 1962). During REM sleep, somatic motoneurons in the anterior horn (AH) of the spinal cord were shown to be hyperpolarized (Chase et al., 1989; Soja et al., 1991; Brooks and Peever, 2011) through inhibition by glycinergic neurons in the ventromedial medulla (VMM; Gly ${ }^{\text {VM }}$ neurons; Holstege and Bongers, 1991; Morales et al., 2006; Vetrivelan et al., 2009; Lai et al., 2010). However, it is still not clear whether there is a specific population of Gly ${ }^{\mathrm{VMM}}$ 
neurons that are specialized to induce REM-atonia, and the mechanism that regulates these REM-atonia inducing neurons.

REM sleep behavior disorder (RBD) is thought to be caused by dysfunction of REM-atonia-generating circuits (Peever et al., 2014). RBD patients exhibit motor behaviors, such as kicking, punching, falling out of bed, standing, jumping, running, grabbing, talking, screaming, yelling, and inordinate jerking of the body and limbs, which might reflect dream content while they are in an REM sleep state (Schenck et al., 1986; Iranzo et al., 2016). Precise understanding of the circuits that evoke REM-atonia is a necessary step to know the pathophysiology of RBD.

Another important REM sleep-related abnormality is cataplexy, a symptom of narcolepsy type 1, which is caused by degenerative loss of hypothalamic neurons that produce neuropeptides orexin A and orexin B (Nishino et al., 2000; Peyron et al., 2000; Thannickal et al., 2000). It manifests as sudden weakening of postural muscle tone triggered by strong positive emotions such as laughter, joking, and delight (Scammell, 2015). Mice lacking orexin peptides, orexin neurons, or orexin receptors recapitulate human narcolepsy type 1 phenotypes (Chemelli et al., 1999; Hara et al., 2001; Willie et al., 2003; Sakurai, 2007). During the dark period, these narcoleptic mice sometimes exhibit cataplexylike abrupt behavioral arrest with muscle atonia that manifests as direct transitions from wakefulness to REM sleep in electroencephalogram/electromyogram (EEG/EMG) recordings. Generally, cataplexy is interpreted as a pathologic intrusion of REM-atonia during wakefulness. However, the precise neural circuits regulating REM-atonia and cataplexy, and whether these pathways are common or distinct have been unknown.

To address these questions, we first explored central neural circuits regulating REM-atonia at the cellular level. We found the existence of a specific population of Gly ${ }^{\mathrm{VMM}}$ neurons that send innervation exclusively to somatic motoneurons. We found that these neurons have a different input-output structure from that of other Gly ${ }^{\mathrm{VMM}}$ neurons. Inhibition of these neurons or of input from the sublaterodorsal tegmental nucleus (SLD) resulted in REM sleep without atonia. The same manipulations in narcoleptic orexin-ataxin-3 mice reduced the total time, duration, and episode number of cataplexy. Our data demonstrate the existence of a population of Gly ${ }^{\mathrm{VMM}}$ neurons that play an important role in inducing REM-atonia and cataplexy.

\section{Materials and Methods}

\section{Animals}

Orexin-ataxin3 (Hara et al., 2001), Ai9 (RCL-tdT; Madisen et al., 2010), and vGlut2-iresCre (Vong et al., 2011) mice were described previously. Twelve- to 20-week-old male mice (backcrossed to C57BL/6J) were used. GlyT2-iCre mice were generated by homologous recombination in C57BL/6N embryonic stem cells and implantation in eight-cell-stage embryos (ICR). A targeting vector was designed to insert an iCre and $p g k$-Neo cassette in exon 2 of the GlyT2 gene so that the endogenous GlyT2 promoter drives expression of $i$ Cre (Fig. 1A). Chimeric mice were crossed with C57BL/6J females (The Jackson Laboratory). The Pgk-Neo cassette was removed by crossing them with FLP66 mice (Takeuchi et al., 2002), which had been backcrossed to C57BL/6J mice at least 10 times. Initially, F1 hybrids from mating heterozygotes with heterozygotes were generated. We backcrossed them to C57BL/6J mice at least eight times.

All experiments were performed on GlyT2-iCre heterozygotes. Mice were maintained under a strict $12 / 12 \mathrm{~h} \mathrm{light/dark} \mathrm{cycle} \mathrm{in} \mathrm{a} \mathrm{tempera-}$ ture-controlled and humidity-controlled room and fed ad libitum. All experimental procedures involving mice were approved by the Animal Experiment and Use Committee of Tsukuba University and were thus in accordance with $\mathrm{NIH}$ guidelines.
Plasmids

pAAV-EF1a-FLEX(loxP)-EYFP-WPRE-HGHpA (\#20 296), pcDNASADB19L (\#32632), pcDNA-SADB19G (\#32633), pcDNA-SADB19N (\#32 630), pcDNA-SADB19P (\#32 631), and pSADdeltaG-GFP-F2 (\#32 635) were obtained from Addgene. $p A A V$-CAG-FLEX(Frt)-TVA-mCherry and $p A A V$-CAG-FLEX(Frt)-RG (rabies glycoprotein; RG) were provided by Naoshige Uchida (Harvard University). pAAV-hSyn-FLEX(loxP)-TeNTLC$P 2 A-E Y F P$ was provided by Katsuyasu Sakurai (University of Tsukuba). pAAV-CAG-FLEX(Frt)-TeNTLC-P2A-EYFP and pAAV-CAG-FLEX(Frt)EYFP were generated by swapping the TVA-mCherry cassette of $p A A V$ CAG-FLEX(Frt)-TVA-mCherry with TeNTLC-EYFP or EYFP. AAV8hSyn-FLEX(loxP)-hM3Dq-mCherry (\#44 361-AAV8) was obtained from Addgene.

\section{Virus}

AAV vectors were packed with pHelper (Stratagene) and $p R C 2$ mi342 (Takara) or pAAV2-rh10 (provided by Penn Vector Core) using a triple transfection, helper-free method. CAV2-FLEX(loxP)$F l p$ virus was obtained from Biocampus Montpellier. $S A D \triangle G-G F P$ (EnvA) was made by transfecting pcDNA-SADB19L, pcDNA-SADB19G, pcDNA-SADB19N, pcDNA-SADB19P, and pSADdeltaG-GFP-F2 in B7GG cells, followed by pseudotyping in BHK-RGCD-EnvA cells, and ultracentrifugation (Osakada and Callaway, 2013). The titers of recombinant AAV vectors (genomic copies $/ \mathrm{ml}$ ) were as follows; AAV10-CAG-FLEX (Frt)-TVA-mCherry, $4.0 \times 10^{13}$; AAV10-CAG-FLEX(Frt)-RG, $1.0 \times$ $10^{13} ; \quad A A V 10-E F 1 a-F L E X(l o x P)-E Y F P, \quad 5.8 \times 10^{13} ;$ AAV8-hSynFLEX(loxP)-hM3Dq-mCherry, $2.0 \times 10^{13} ;$ AAV2-hSyn-FLEX(loxP)TeNTLC-P2A-EYFP, $2.6 \times 10^{13}$; AAV10-CAG-FLEX(Frt)-EYFP, $2.8 \times 10^{13}$; $A A V 2-C A G-F L E X(F r t)-T e N T L C-P 2 A-E Y F P, \quad 3.5 \times 10^{12} ; S A D \Delta G-$ $\operatorname{GFP}(\operatorname{Env} A), 4.2 \times 10^{8}$ (infectious unit/ml).

\section{Surgery}

Mice were deeply anesthetized with isoflurane (1-2\%) for all procedures. They were positioned in a stereotaxic frame (David Kopf Instruments). The coordinates and volumes used in this study were as follows: VMM: AP, $-6.2 \mathrm{~mm}$; ML, $-0.4 \mathrm{~mm}$ from bregma; $\mathrm{DV},-5.3 \mathrm{~mm}$ from brain surface; SLD: AP, $-5.0 \mathrm{~mm}$; ML, $-0.75 \mathrm{~mm}$ from bregma; DV, $-3.5 \mathrm{~mm}$ from brain surface; $120 \mathrm{nl}$ in each site. For anterograde tracing of Gly ${ }^{\mathrm{VMM}}$ neurons, we injected AAV8-hSyn-FLEX(loxP)-hM3Dq-mCherry into the VMM of GlyT2-iCre mice (Fig. $2 A$ ) with a glass microcapillary syringe using an air pressure injector system (Picospritzer III, Parker).

For cTRIO experiments (Fig. 3), the back skin around Th12-L5 of GlyT2-iCre mice was incised using clippers. Then, mice were placed in spinal cord clamps (Pro Device Instruments), and the paraspinal muscles and connective tissues were removed to expose the intervertebral foramen between Th13 and L1 vertebrae. Meninges were carefully removed using surgical forceps and the tip of a $27-\mathrm{G}$ needle. Then, a glass microcapillary syringe was inserted into the left $\mathrm{AH}$ of the spinal cord of $\mathrm{L} 1$ (700 $\mu \mathrm{m}$ lateral to midline and $900 \mu \mathrm{m}$ deep to surface of spinal cord), and $120 \mathrm{nl} C A V 2-F L E X(l o x P)-F L P$ was injected using an air pressure injector system (Picospritzer III, Parker) over $5 \mathrm{~min}$. Then, mice were placed in a stereotaxic frame under anesthesia, and a cocktail of $A A V 10$ CAG-FLEX(Frt)-TVA-mCherry and AAV10-CAG-FLEX(Frt)-RG (120 $\mathrm{nl}$, mixed at a 1:2 ratio) was injected into the left VMM. Two weeks after the injection, $0.12 \mu \mathrm{l} S A D \Delta G-G F P(E n v A)$ was injected into the same region. Mice were kept in home cages for $7 \mathrm{~d}$ before perfusion. For silencing Gly ${ }^{\mathrm{VMM}}$, we injected AAV2-hSyn-FLEX(loxP)-TeNTLC-P2AEYFP or AAV10-EF1a-FLEX(loxP)-EYFP into the VMM of GlyT2-iCre mice or GlyT2-iCre; orexin-ataxin 3 mice (Fig. $5 A, 6 A$ ). For pathway specific manipulation, we injected CAV2-FLEX(loxP)-FLP into the VMM and AAV2-CAG-FLEX(Frt)-TeNTLC-P2A-EYFP or AAV10-CAG-FLEX (Frt)-EYFP into the SLD of vGlut2-iresCre mice or vGlut2-iresCre; orexin-ataxin 3 mice (Fig. 5I, 6I). EEG and EMG electrodes were implanted into the skull of each mouse. Two pins of electrode for EEG recording were placed $0.15 \mathrm{~mm}$ posterior to bregma, $0.60 \mathrm{~mm}$ right lateral, and $2.65 \mathrm{~mm}$ posterior to bregma/ $0.60 \mathrm{~mm}$ right lateral. Stainless steel wires (AS633, Cooner Wire) as electrodes for EMG were inserted bilaterally into the neck muscles of each mouse, and each electrode was attached to the skull using dental cement (3M, 56818). Mice were housed singly for recovery after surgery. 


\section{EEG/EMG recording}

Mice were habituated to the recording conditions for another week and then recorded for two consecutive 24-h periods. Averages of each time on two recording days were used as raw data, and data from all individual animals used in these studies were used to determine their sleep/wakefulness characteristics. EEG/EMG signals were amplified and filtered (AB-611J, Nihon Koden, EEG: $0.5-64 \mathrm{~Hz}$, EMG: $16-64 \mathrm{~Hz})$, digitized at a sampling rate of $128 \mathrm{~Hz}$, and recorded using EEG/ EMG recording software (Vital recorder, Kissei Comtec). EEG/EMG signals were scored by characterizing 10-s epochs in wakefulness, non-REM (NREM) sleep, REM sleep, or cataplexy-like episodes (CLEs). In this study, we defined direct transitions from wakefulness to REM sleep as CLEs as described in a previous study (Scammell et al., 2009). Although muscle tone during REM sleep in some cases in this study was pathologic, the EEG pattern in REM sleep could be clearly distinguished from that in wakefulness by EEG. We scored each epoch as REM sleep only when the $\delta$ /theta ratio was below 1.0 after NREM sleep. EMG voltage amplitudes were recorded in every $4 \mathrm{~ms}$ as a raw value, and 2500 raw values in 10-s epochs were averaged (mean value). Then, the absolute values of the difference between the raw value and mean value in each $4 \mathrm{~ms}$ were averaged in each 10-s epoch (epoch value). Finally, the averages of epoch value in each stage were calculated (mean EMG integral). We normalized mean EMG integral in REM sleep by that in NREM sleep to normalize variations of EMG electrode positions.

\section{Immunohistochemistry (IHC)}

Animals were deeply anesthetized with isoflurane, and then perfused transcardially with $10 \%$ sucrose in PBS, followed by $4 \%$ paraformaldehyde in PBS (4\% PFA). Brains were removed and postfixed overnight in $4 \% \mathrm{PFA}$ at $4^{\circ} \mathrm{C}$ and transferred to $30 \%$ sucrose in PBS at $4^{\circ} \mathrm{C}$. After overnight incubation, brains were frozen in Tissue-Tek O.C.T. Compound (Sakura) and stored at $-80^{\circ} \mathrm{C}$. Coronal brain sections were sliced at $80 \mu \mathrm{m}$ using a cryostat (Leica Biosystems).

The serial brain sections were collected in 12well plates and washed with PBS three times. The sections were immersed in $1 \%$ Triton in PBS at room temperature for $3 \mathrm{~h}$. Then, sections were blocked with 10\% BSA (Blocking One, Nacalai Tesque) in PBS with $0.3 \%$ Triton X-100 (blocking solution) at room temperature for $1 \mathrm{~h}$. The sections were incubated with first antibody in blocking solution at $4^{\circ} \mathrm{C}$ overnight. The sections were rinsed three times with PBS followed by secondary antibody treatment at $4^{\circ} \mathrm{C}$ overnight. Sections were counterstained with Nissl (1:500; Thermo Fisher Scientific catalog \#N21479) or 4',6-diamidino-2-phenylindole (DAPI; Thermo Fisher Scientific catalog \#D3571, RRID: AB 2307445). After incubation, sections were rinsed with PBS, mounted and coverslipped. The primary antibodies used in this study were: rat anti-green fluorescent protein (1:1000; Nacalai Tesque catalog \#04404-84, RRID: AB_10013361), goat antimCherry (1:1000; SICGEN catalog \#AB0040-200, RRID: AB_2333092), rabbit anti-red fluorescent protein (1:1000; Rockland catalog \#600-401-

D
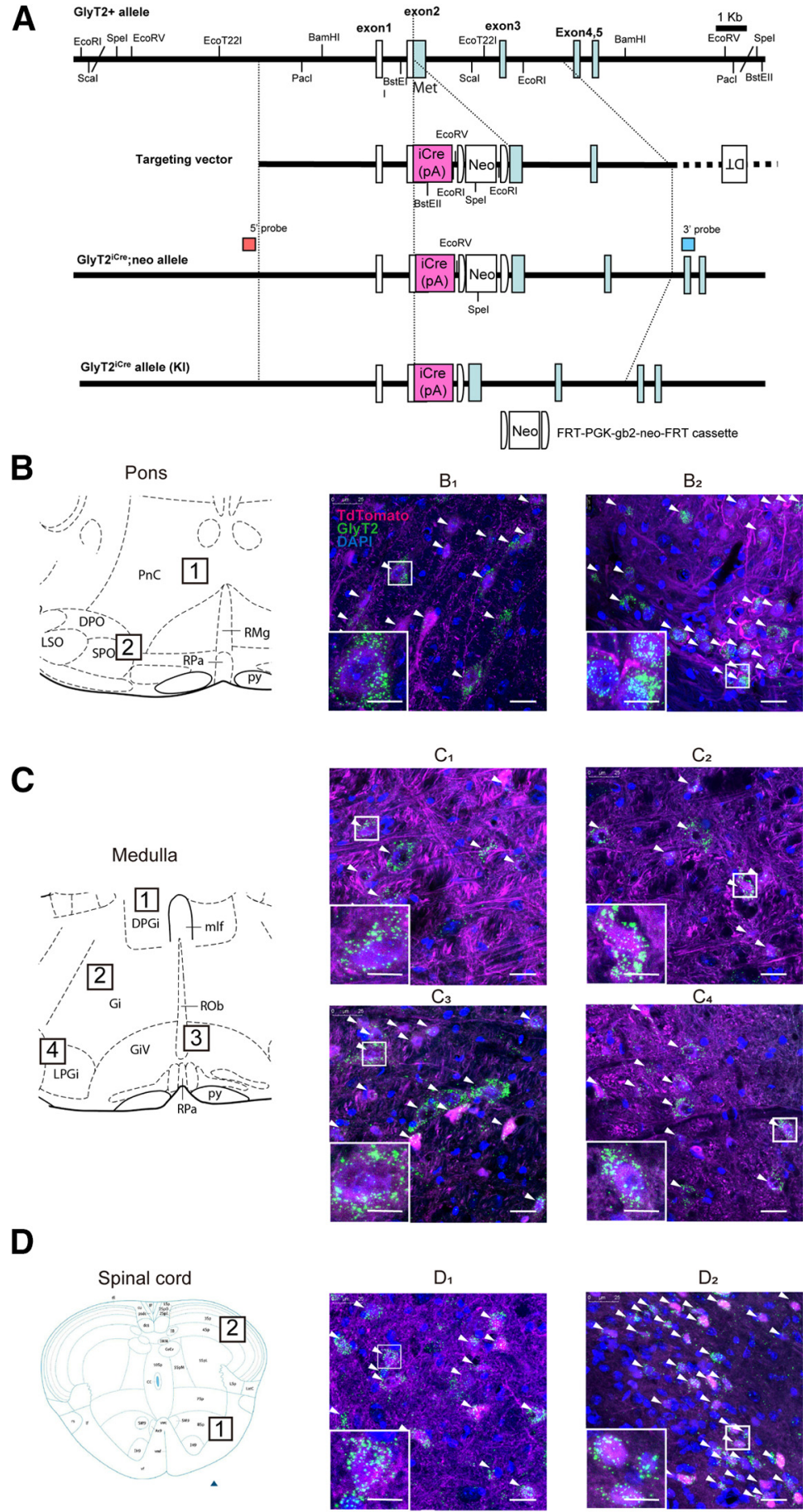

Figure 1. Generated mice specifically expressing iCre recombinase in glycinergic neurons. $A$, Targeting strategy for GlyT2-iCre mice. $\boldsymbol{B}-\boldsymbol{D}$, Specific expression of TdTomato by GlyT2-positive neurons in the pons $(\boldsymbol{B})$, medulla $(\boldsymbol{C})$, and spinal cord (D) of GlyT2-iCre;Ai9 transgenic mice. Brain sections were stained by in situ hybridization with GlyT2 RNA probe. Histochemical images of designated regions shown as rectangles in $\mathbf{B}-\mathbf{D}$ are shown in $\mathbf{B 1}, \mathbf{B 2}, \mathbf{C 1}-\mathbf{C} \mathbf{A}, \mathbf{D 1}, \mathbf{D 2}$. Scale bars: $25 \mu \mathrm{m}$. Lower left insets show high-power images of the boxed area. Scale bars: $10 \mu \mathrm{m}$. The brain atlases were taken from the Paxinos mouse brain atlas, and the spinal cord atlas was taken from Allen Brain Atlas (https://mousespinal.brain-map. org/imageseries/showref.html). DPGi, dorsal paragigantocellular nucleus; DPO, dorsal periolivary region; mlf, medial longitudinal fasciculus; Gi, gigantocellular reticular nucleus; GiV, gigantocellular reticular nucleus, ventral part; LPGi, lateral paragigantocellular nucleus; LSO, lateral superior olive; PnC, pontine reticular nucleus, caudal part; py, pyramidal tract; RMg, raphe magnus nucleus; $\mathrm{ROb}$, raphe obscurus nucleus; $\mathrm{RPa}$, raphe pallidus nucleus; $\mathrm{SPO}$, superior paraolivary nucleus.

379, RRID: AB_2209751), goat anti-choline acetyltransferase (1:500; Millipore catalog \#AB144P, RRID:AB_2079751), rabbit anti-tryptophan hydroxylase 2 (1:500; Abcam catalog \#ab111828, RRID:AB_ 10862137), and mouse anti-tyrosine hydroxylase (1:2000; Santa Cruz 
Table 1. Colocalization of neurons expressing GlyT2 mRNA and TdTomato in brainstem and spinal cord region

\begin{tabular}{|c|c|c|}
\hline Brain region (number of animals) & GlyT2 mRNA-expressing neurons in TdTomato-expressing neurons & TdTomato-expressing neurons in GlyT2 mRNA-expressing neurons \\
\hline $\mathrm{Pn} 0 / \mathrm{PnC}(n=2)$ & $97.2 \%$ (106/109 cells) & $99.1 \%(106 / 107$ cells $)$ \\
\hline SPO/RPO/LSO/MVPO/LVPO $(n=2)$ & $97.6 \%(123 / 126$ cells) & $99.2 \%$ (123/124 cells) \\
\hline $\mathrm{GiA} / \mathrm{GiV}(n=3)$ & $95.3 \%(285 / 299$ cells $)$ & $97.9 \%(285 / 291$ cells $)$ \\
\hline $\mathrm{Gi}(n=3)$ & $95.5 \%$ (126/132 cells) & $99.2 \%$ (126/127 cells) \\
\hline DPGi $(n=2)$ & $95.8 \%$ (46/48 cells) & $100 \%$ (48/48 cells) \\
\hline LPGi $(n=2)$ & $96.2 \%$ (126/131 cells) & $100 \%(126 / 126$ cells $)$ \\
\hline Renshaw cells in sp7 and sp8 $(n=2)$ & $91.7 \%$ (121/132 cells) & $100 \%(121 / 121$ cells $)$ \\
\hline Dorsal horn neurons in sp3 and sp4 $(n=2)$ & $93.8 \%(226 / 241$ cells $)$ & $98.7 \%(226 / 229$ cells) \\
\hline Total $(n=3)$ & $94.1 \%(1288 / 1369$ cells) & $99.1 \%(1288 / 1300$ cells $)$ \\
\hline
\end{tabular}

A

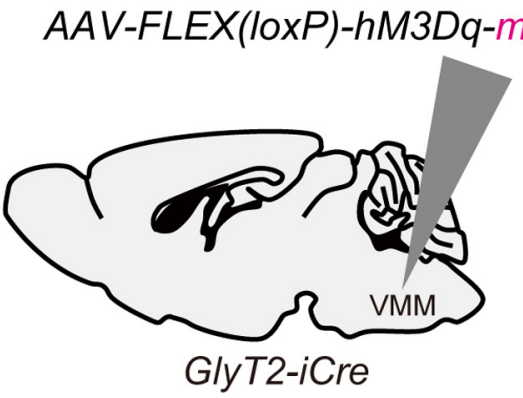

C
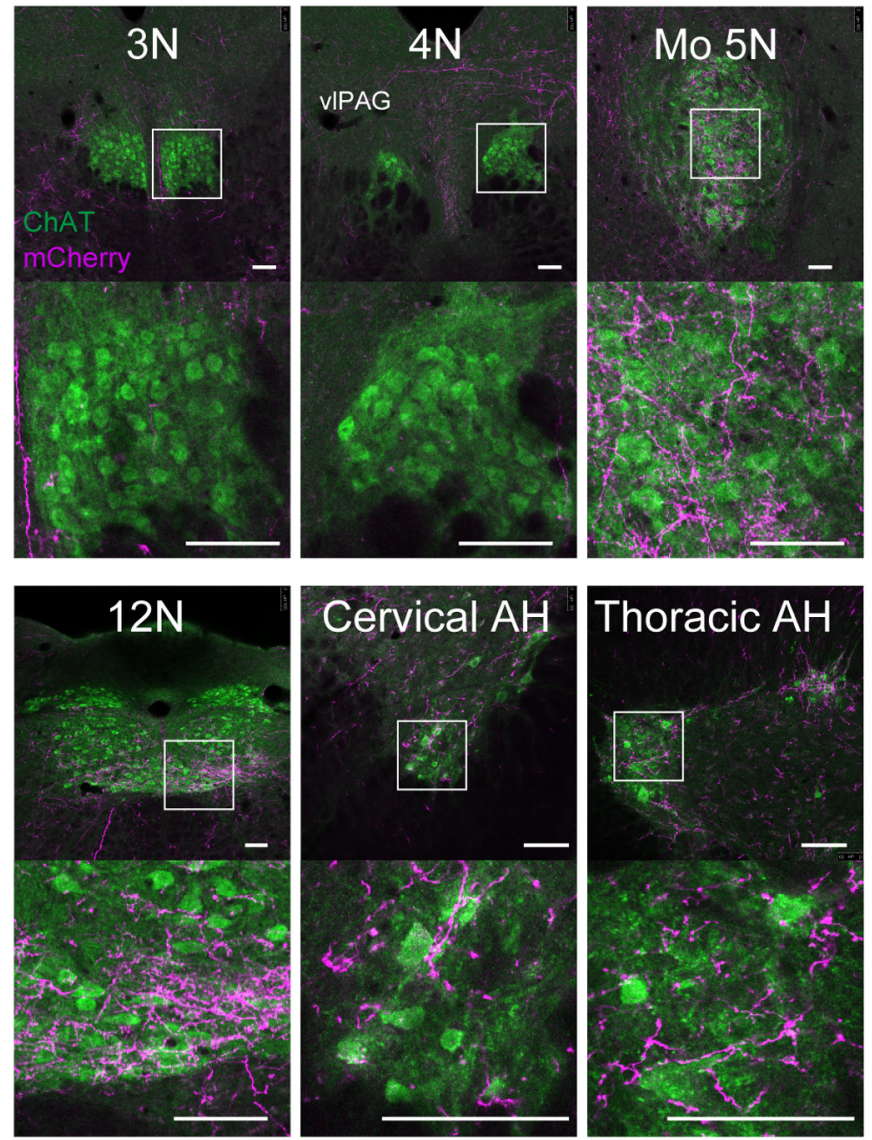

B
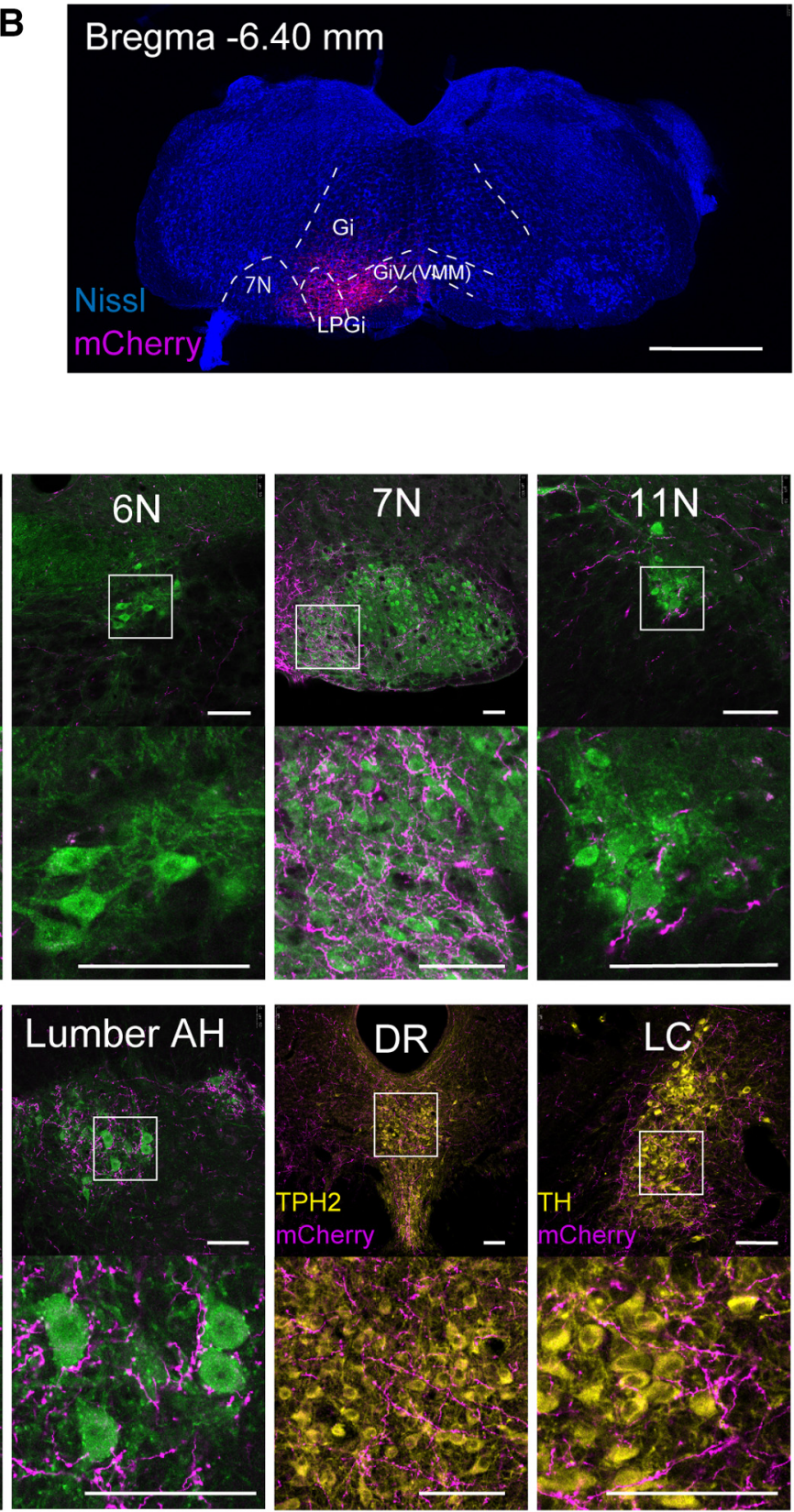

Figure 2. Axonal projections of Gly ${ }^{\mathrm{VMM}}$ neurons. $\boldsymbol{A}$, Schematic of AAV injection. AAV8-hSyn-FLEX(IoxP)-hM3Dq-mCherry was injected unilaterally into the VMM of GlyT2-iCre mice. $\boldsymbol{B}$, Representative image of mCherry-positive neurons. Scale bar: $1 \mathrm{~mm}$. $\boldsymbol{C}$, Representative images show mCherry-positive axonal fibers (magenta) in brain regions and spinal cord. ChAT-positive neurons are stained (green). TPH2 and TH are stained to characterize serotonergic neurons in the dorsal raphe and noradrenergic neurons in the locus coeruleus (yellow). Scale bars: $100 \mu \mathrm{m}$. 
Table 2. Projection sites from Gly ${ }^{\mathrm{VMM} \rightarrow \mathrm{AH}^{\mathrm{H}}}$ neurons and Gly ${ }^{\mathrm{VMM}}$ neurons

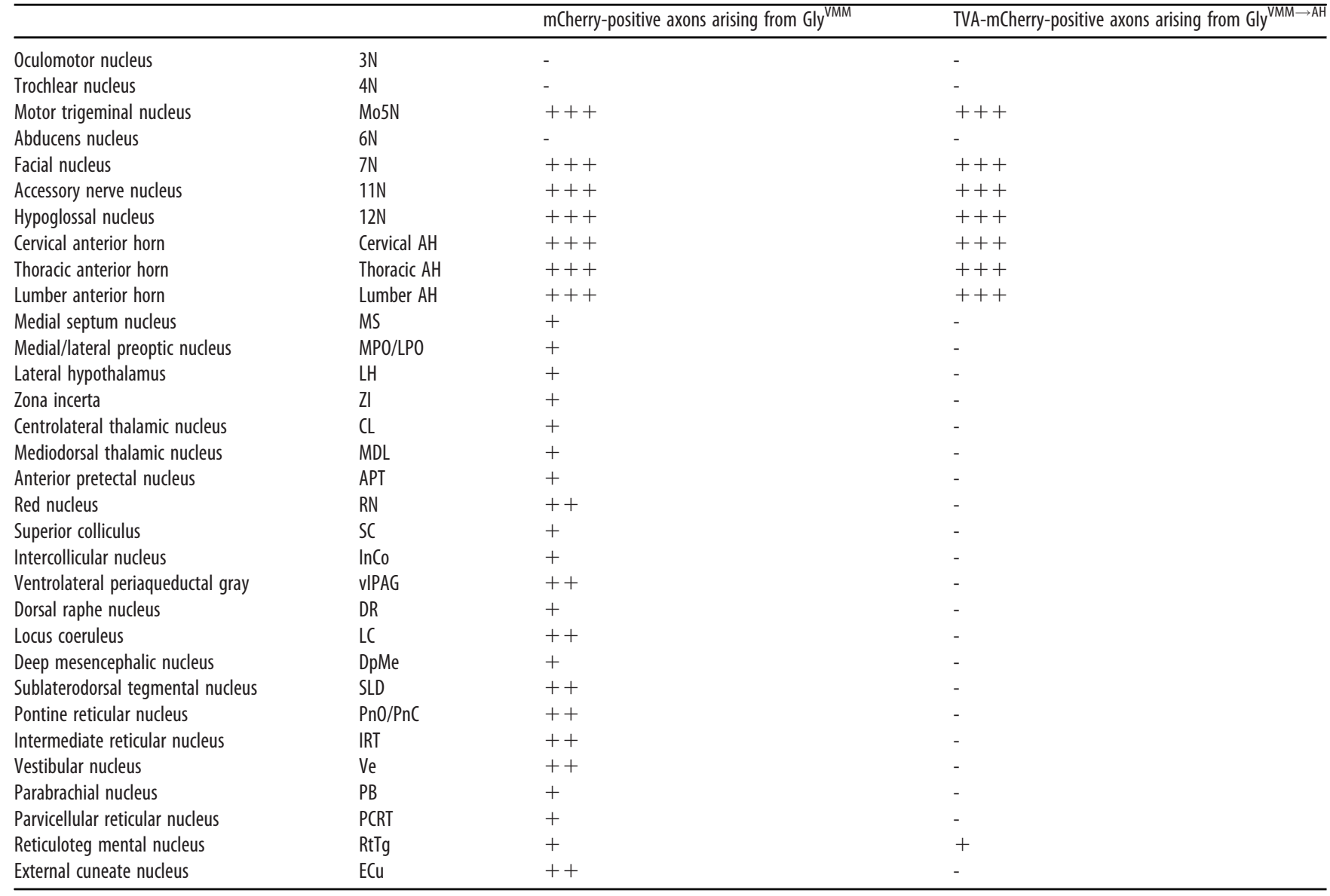

- , no recognizable fibers; + , slightly dense fibers; ++ , moderately dense fibers; +++ , highly dense fibers.

Biotechnology catalog \#sc-25269, RRID: AB_628422). The secondary antibodies were: Alexa Fluor 488 donkey anti-rat (1:1000; Thermo Fisher Scientific catalog \#A-21208, RRID: AB_2535794), Alexa Fluor 488 donkey anti-mouse (1:1000; Thermo Fisher Scientific catalog \#A21202, RRID:AB_141607), Alexa Fluor 488 donkey anti-rabbit (1:250; Thermo Fisher Scientific catalog \#A-21206, RRID:AB_2535792), Alexa Fluor 594 donkey anti-goat (1:1000; Thermo Fisher Scientific catalog \#A11058, RRID:AB_2534105), Alexa Fluor 594 donkey anti-rabbit (1:1000; Thermo Fisher Scientific catalog \#A-21207, RRID:AB_141637), and Alexa Fluor 647 donkey anti-goat (1:1000; Abcam catalog \#ab150135, RRID: AB_2687955).

Representative images were obtained with a confocal microscope (Zeiss LSM 800 or Leica SP8).

Fluorescence in situ hybridization (FISH)

To detect GlyT2, ChAT and Vglut2 mRNA, FISH was performed using a kit according to the manufacturer's instructions (RNAscope Fluorescent Multiplex Reagent kit, catalog \#320850, Advanced Cell Diagnostics). After fixation and cryoprotection, brains and spinal cords were frozen in Tissue-Tek O.C.T. Compound (Sakura) and stored at $-80^{\circ} \mathrm{C}$. Coronal brain sections were sliced at $20 \mu \mathrm{m}$ using a cryostat (Leica Biosystems). We used a GlyT2 probe (SLC6A5-C1, 409741-C1), ChAT probe (ChAT-C1, 408731-C1) and Vglut2 probe (SLC17A6-C2, 540961C2) for hybridization. Fluorescence images were obtained using a confocal microscope (Leica Biosystems, SP8).

Data acquisition and analyses for whole-brain tracing

For retrograde tracing analysis, we counted GFP-single positive input neurons in every third $80-\mu \mathrm{m}$ section of the whole brain based on the mouse brain map by Franklin and Paxinos (2001). As total number of input neurons varied among brains, we calculated the percentage of input neuron number in each area of the total number of input neurons counted in the same brain, as previously described (Weissbourd et al., 2014). Based on previous studies (Weber et al., 2018; Erickson et al., 2019), we termed the ventral part of the laterodorsal tegmental nucleus the SLD in this study.

\section{Axon signal quantification}

To compare the projection patterns of $\mathrm{Gly}^{\mathrm{VMM}}$ and $\mathrm{Gly}^{\mathrm{VMM} \rightarrow \mathrm{AH}}$, we quantified mCherry-positive axon density in each brain area using imageJ software. A boxed area $\left(200 \times 200 \mu \mathrm{m}^{2}\right)$ for the mCherry channel of each brain region was cropped, converted to an eight-bit image, and binarized by thresholding. We manually determined the threshold value that captured axons in the motor trigeminal nucleus (Mo5N) in each mouse. The threshold value was kept constant for all sections within a brain and spinal cord. Then, the area fraction above the threshold was measured in each region, and the percentage fraction of each region normalized by the sum of fractions was calculated in each mouse.

\section{Statistics}

All results are expressed as mean \pm SEM. Analysis of significance was performed using two-tailed, unpaired $t$ test for single variables. Welch's $t$ test was used when the variances of two group means were different. Two-way ANOVA followed by Bonferroni post hoc tests was used to analyze hourly plots of sleep/wake cycles and compare the percentage of input neurons in each brain region between cTRIO tracing and conventional tracing (Fig. 4G). All statistical analyses were performed using GraphPad Prism 8.0. Differences were considered significant at $* p<0.05, * * p<0.01$, and $* * * p<0.001$. 


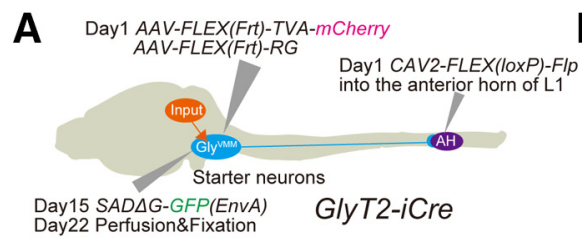

B Day22 Perfusion\&Fixation

C Mouse ID \#4- \#50 \#60 \#70

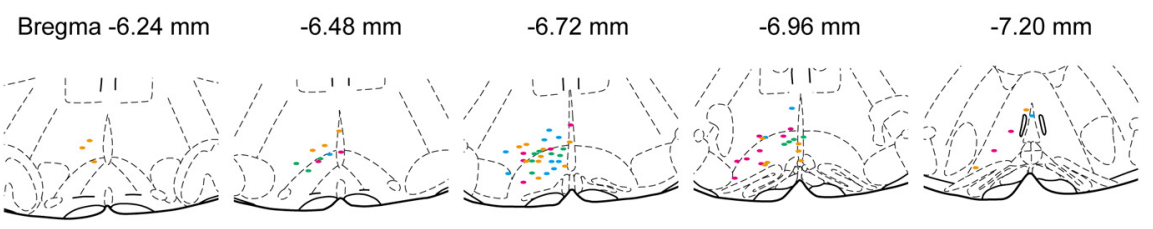

D
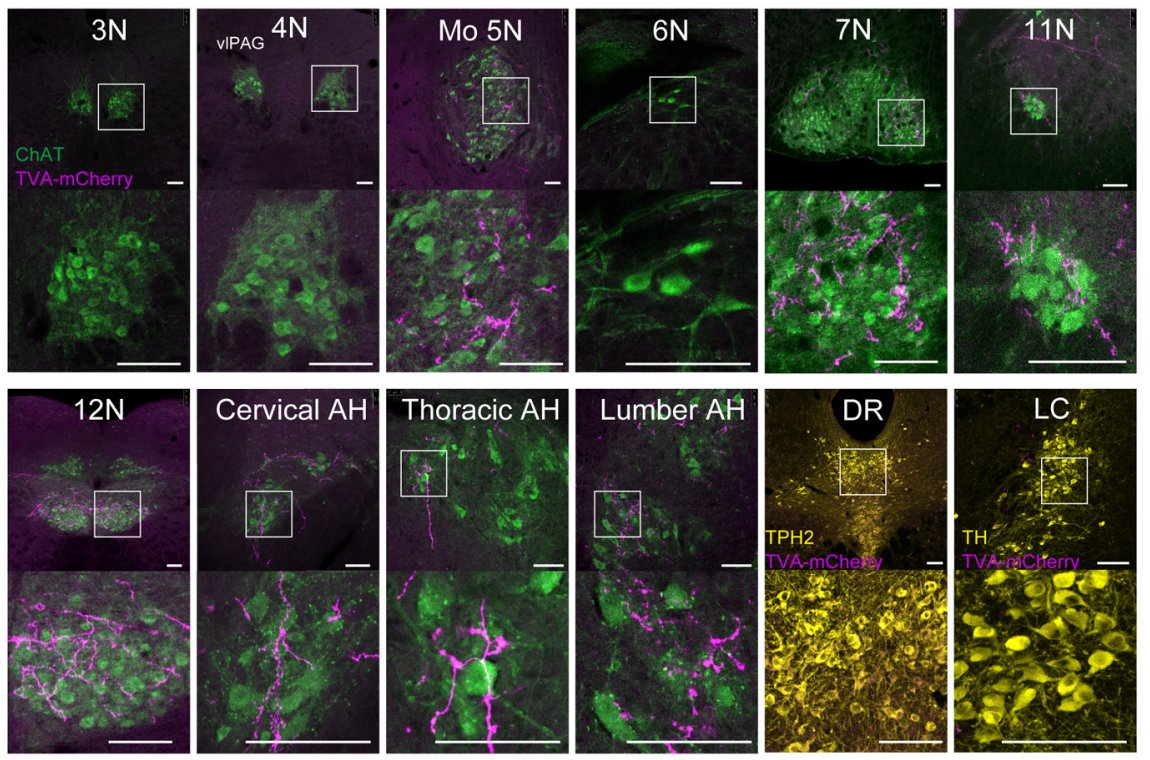

$\mathrm{E}$

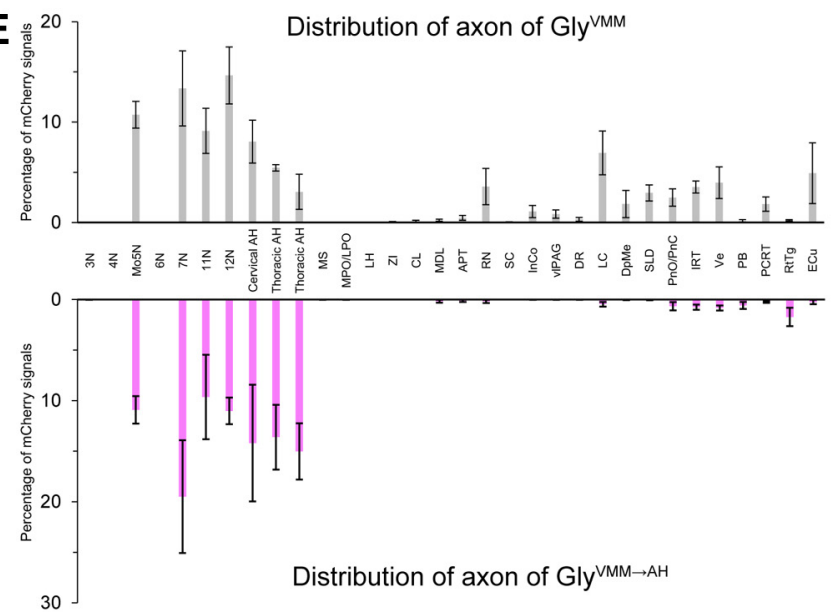

Figure 3. Axonal projections of Gly ${ }^{\mathrm{VMM} \rightarrow \mathrm{AH}}$ neurons. A, Experimental procedure of cTRI0 study. We injected CAV2FLEX(loXP)-FIp into the AH of the spinal cord (L1) and AAV-FLEX(Frt)-TVA and AAV-FLEX(Frt)-RG into the VMM (day 1). SAD $\triangle G$-GFP(EnvA) was injected into the VMM (day 15). $\boldsymbol{B}$, Representative images of starter cells in VMM. Upper panel, Low-power image. Lower panel, High-power image. Nissl is stained blue, TVA-mCherry is stained magenta, and GFP is stained green. Scale bar: $1 \mathrm{~mm}$ (upper), $100 \mu \mathrm{m}$ (lower). C. Schematic drawings showing location of starter neurons in VMM. D, TVA-mCherry-positive axons in the brainstem and spinal cord. ChAT is stained green, and TPH2 or TH is stained green, while TVA-mCherry is stained magenta. $\boldsymbol{E}$, Percentage of total mCherry axon arborization of Gly ${ }^{\mathrm{VMM}}$ neurons (upper) and Gly ${ }^{\mathrm{VMM} \rightarrow \mathrm{AH}}$ neurons (below) across brain and spinal cord regions ( $n=4$ in each group). $\boldsymbol{F}$, Comparison of $\mathrm{mCherry}$ fraction in motoneurons between direct Gly ${ }^{\mathrm{VMM}}$ labeling and projection-specific Gly ${ }^{\mathrm{VMM} \rightarrow \mathrm{AH}}$ labeling; $* p<0.05$. Unpaired $t$ test. APT, anterior pretectal nucleus; $\mathrm{CL}$, centrolateral thalamic nucleus; ECu, external cuneate nucleus; InCo, intercollicular nucleus; IRT, intermediate reticular nucleus; LPO, lateral

\section{Results}

A population of glycinergic neurons in the ventral medulla sends projections almost exclusively to somatic motoneurons

Glycinergic and GABAergic inhibitory neurons in the ventral medulla have been implicated in regulation of locomotion by regulating motor function (O'Brien and Berger, 1999; Goulding, 2009; Stanek et al., 2014; Capelli et al., 2017). Recent studies suggested that glycinergic/GABAergic neurons in the VMM also mediate postsynaptic inhibition of motoneurons to induce REM-atonia and GlyT2-positive neurons projecting to the spinal cord are activated during REM sleep (Garcia et al., 2018). It has been unclear whether there is a specific population of glycinergic neurons in the VMM that are involved in induction of REM-atonia.

Glycine acts as an inhibitory neurotransmitter in the brainstem and spinal cord, regulating a variety of physiological functions. There are two types of glycine transporters, GLYT1 and GLYT2, which transport extracellular glycine to the intracellular space (Eulenburg et al., 2005). While GLYT1 eliminates glycine from the synaptic cleft, GLYT2 is essential for glycine uptake from the extracellular space to the cytoplasm. GLYT1 is predominantly expressed in brain glial cells, whereas GLYT2 is specifically expressed in glycinergic neurons and is considered to be a specific marker of glycinergic neurons (Poyatos et al., 1997).

To specifically manipulate the function of glycinergic neurons, we generated mice in which glycinergic neurons express codon-improved Cre recombinase (iCre) by knocking-in iCre in the GlyT2 gene (GlyT2-iCre mice; Fig. 1A). To explore expression of Cre, we crossed GlyT2-iCre mice with $A i 9$ (RCL-tdT) mice, which have a loxP-flanked transcription blocking cassette preventing transcription of a CAG promoter-driven tdTomato inserted into the Gt(ROSA)26Sor locus. Specific expression of Cre activity in GlyT2-positive neurons in the brain stem and spinal cord regions were confirmed by in situ hybridization histochemistry (ISH) using brain slices prepared from GlyT2-iCre;Ai9 mice (Fig. $1 B-D$ ). This study suggested that

preoptic nucleus; MDL, mediodorsal thalamic nucleus; MP0, medial preoptic nucleus; MS, medial septum; PB, parabrachial nucleus; $P C R T$, parvicellular reticular nucleus; RtTg, reticulotegmental nucleus of the pons; Ve, vestibular nucleus; Zl, zona incerta. 
glycinergic neurons in the brain stem and spinal cord regions specifically express Cre recombinase, with $94.1 \%$ of tdTomato-positive neurons being positive for GlyT2 mRNA, while $99.1 \%$ of GlyT2-positive neurons expressed tdTomato fluorescence in the brain stem and spinal cord $(n=3$; Fig. $1 B-D$; Table 1 ).

We next injected AAV8-hSyn-Flex (loxP)-hM3Dq-mCherry into the VMM of GlyT2-iCre mice to depict glycinergic neurons in the VMM (Gly ${ }^{\mathrm{VMM}}$ neurons; Fig. $2 A)$. We found abundant expression of mCherry in the VMM (Fig. 2B). Gly ${ }^{\mathrm{VMM}}$ neurons sent mCherry-positive axonal projections to the motor trigeminal nucleus $($ Mo $5 \mathrm{~N})$, facial nerve nucleus $(7 \mathrm{~N})$, accessory nerve nucleus (11N), hypoglossal nerve nucleus $(12 \mathrm{~N})$, red nucleus $(\mathrm{RN})$, dorsal raphe (DR), locus coeruleus (LC), ventrolateral periaqueductal gray (vlPAG), SLD, and pontine reticular nucleus (Pn), but not oculomotor nuclei, such as the oculomotor nerve nucleus $(3 \mathrm{~N})$, trochlear nerve nucleus $(4 \mathrm{~N})$, and abducens nerve nucleus (6N; Fig. 2C; Table 2). mCherrypositive fibers were also found in the $\mathrm{AH}$ at all levels. This observation suggests that Gly ${ }^{\mathrm{VMM}}$ neurons send axonal projections to multiple brain regions including somatic motoneurons.

To understand the mechanism that regulates REM-atonia, it is important to understand the input and output architecture of Gly ${ }^{\mathrm{VMM}}$ neurons that send innervations to motoneurons. To address this, we specifically labeled Gly ${ }^{\mathrm{VMM}}$ neurons that send innervations to the $\mathrm{AH}$ of the spinal cord (Gly ${ }^{\mathrm{VMM} \rightarrow \mathrm{AH}}$ neurons), and further identified upstream input neurons to $\mathrm{Gly}^{\mathrm{VMM} \rightarrow \mathrm{AH}}$ neurons by cTRIO method (Schwarz et al., 2015; Fig. 3A).

We injected a Cre-dependent canine adenoviral vector 2 (CAV2) carrying flippase (Flp; CAV2-FLEX (loxP)-Flp) into the left $\mathrm{AH}$ at the L1 level in GlyT2-iCre mice. Because CAV2 infects axon terminals, and is retrogradely transported to cell bodies, this procedure allowed us to express $\mathrm{Flp}$ specifically in Gly ${ }^{\mathrm{VMM} \rightarrow \mathrm{AH}}$ neurons. We then injected a cocktail of Flp-activatable AAV vectors, AAV10-CAG-FLEX(Frt)TVA-mCherry and AAV10-CAG-FLEX (Frt)-RG, into the left VMM to express TVA and RG in Gly ${ }^{\mathrm{VMM} \rightarrow \mathrm{AH}}$ neurons. Fourteen days later, we injected $S A D \Delta G^{-}$ GFP (EnvA) into the left VMM (Fig. 3A).

As expected, starter cells (TVAmCherry and GFP-double positive neurons) were found in the VMM (Fig. $3 B, C$ ). These neurons were found in the $\alpha$ part of the gigantocellular reticular nucleus (GiA) and ventral part of the gigantocellular

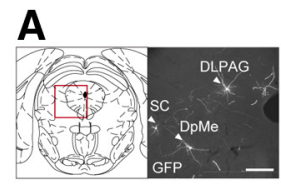

B
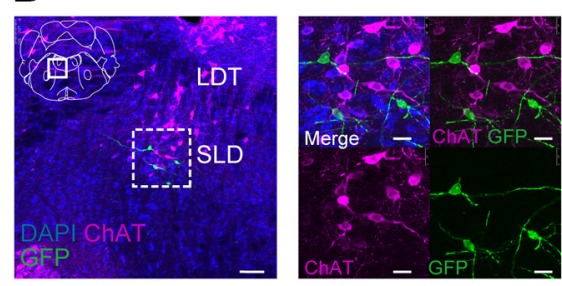

D

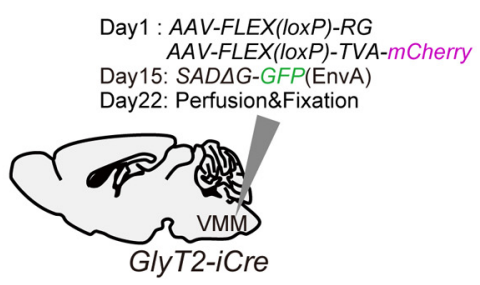

$\mathbf{F}$
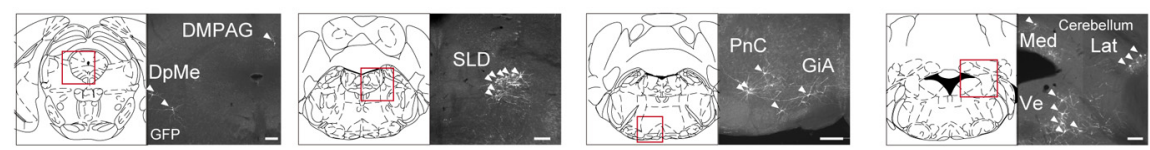

G

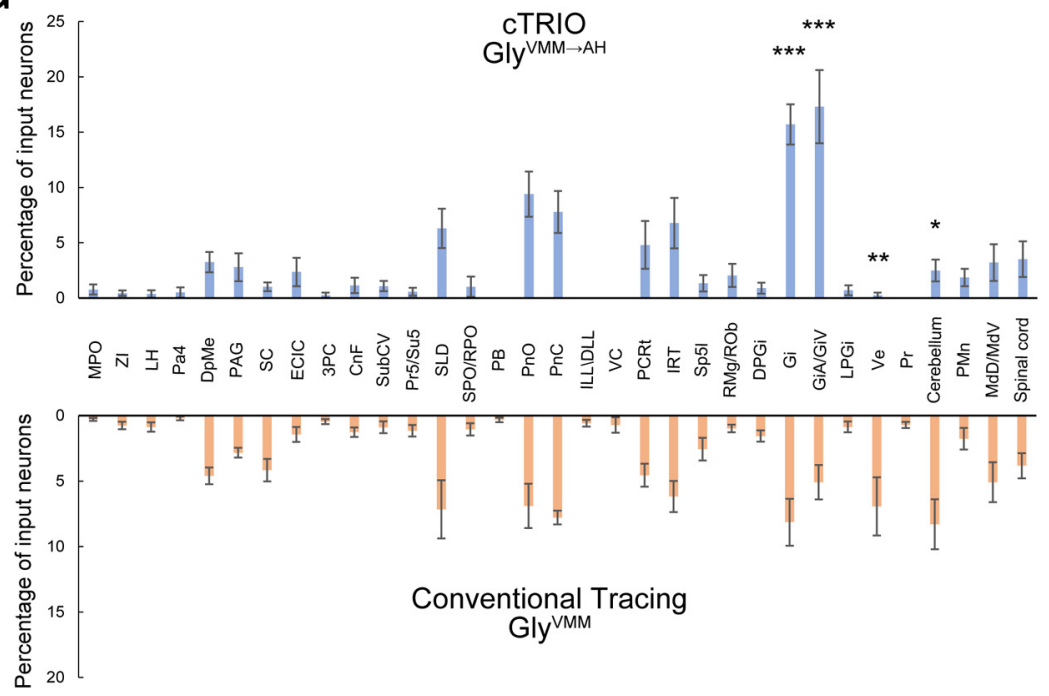

Figure 4. Trans-synaptic retrograde tracings of Gly $\mathrm{VMM} \rightarrow \mathrm{AH}$ and Gly ${ }^{\mathrm{VMM}}$ neurons. $\boldsymbol{A}$, Images show input neurons (white) of Gly ${ }^{\mathrm{VMM} \rightarrow A H}$ revealed by cTRIO (Fig. 3). Scale bar: $200 \mu \mathrm{m}$. B. Fluorescent images of IHC staining for Nissl (blue), GFP (green), and ChAT (magenta) in GFP-positive input neurons in SLD. Left panel, Low-magnification $(20 \times)$ image. Right panels, High-magnification $(63 \times)$ image. Scale bar: $50 \mu \mathrm{m}$ (left), $25 \mu \mathrm{m}$ (right). C, Fluorescent images of in situ hybridization staining for Vglut2 (cyan) and ChAT (magenta) in a GFP-positive input neuron (yellow) in the SLD. Left panel, Low-magnification (20×) image. Right panel, High-magnification $(63 \times)$ image. Scale bar: $50 \mu \mathrm{m}$ (left), $25 \mu \mathrm{m}$ (right). D, Experimental procedure for rabies virus vector tracing. We expressed TVA and RG in glycinergic neurons in the VMM (day 1). SAD $G$ G-GFP (EnvA) was injected into the same site (day 15). $\boldsymbol{E}$, Representative images of starter cells in VMM. TVA-mCherry is stained magenta, while GFP is stained green. Scale bar: $1 \mathrm{~mm}$. $\boldsymbol{F}$, Images show input neurons (white) of Gly ${ }^{\mathrm{VMM}}$ neurons throughout the brain. Scale bar: $200 \mu \mathrm{m}$. $G$, Graphic representation of distribution of input neurons of Gly ${ }^{\mathrm{VMM} \rightarrow A \mathrm{AH}}$ neurons (upper) and Gly ${ }^{\mathrm{VMM}}$ neurons (lower) throughout the brain. Values are mean \pm SEM of four samples. Statistical significance was determined using two-way ANOVA with post hoc Bonferroni's multiple comparison test; $* p<0.05, * * p<0.01, * * * p<0.001$. 3PC, oculomotor nucleus, parvicellular part; CnF, cuneiform nucleus; ECIC, extended cortex of inferior colliculus; DLL, dorsal nucleus of lateral lemniscus; ILL, intermediate nucleus of lateral lemniscus; Lat, lateral cerebellar nucleus; MdV, medullary reticular nucleus, ventral part; Med, medial cerebellar nucleus; Pr5, principal sensory trigeminal nucleus; Pa4, paratrochlear nucleus; PMn, paramedial reticular nucleus; Pn0, pontine reticular nucleus, oral part; PnC, pontine reticular nucleus, caudal part; Pr, prepositus nucleus; RP0, rostral periolivary region; SP5L, spinal trigeminal nucleus, SP0, superior paraolivary nucleus; Su5, supratrigeminal nucleus; SubCV, subcoeruleus nucleus; VC, ventral cochlear nucleus. 
A

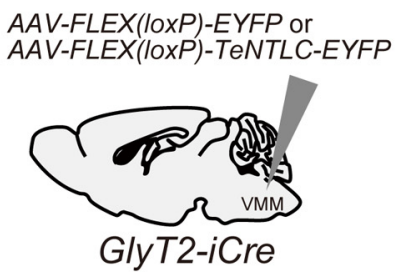

C
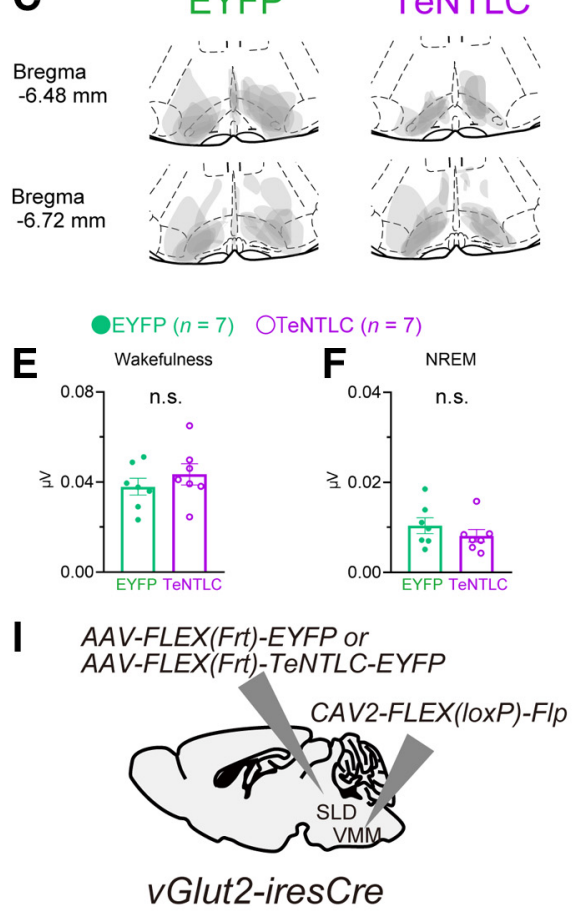

$\mathbf{L}$

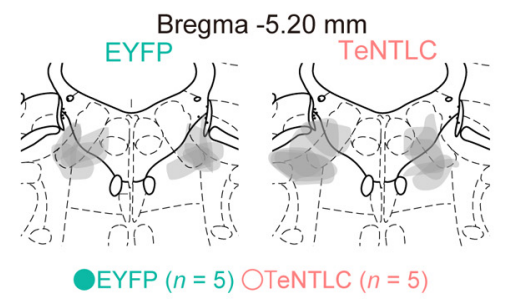

$\mathbf{N}$
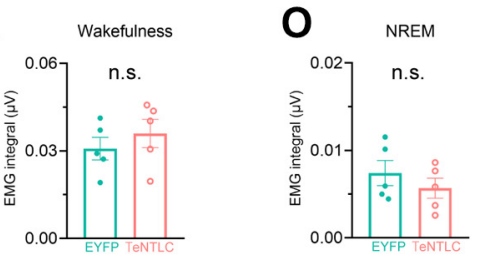

B

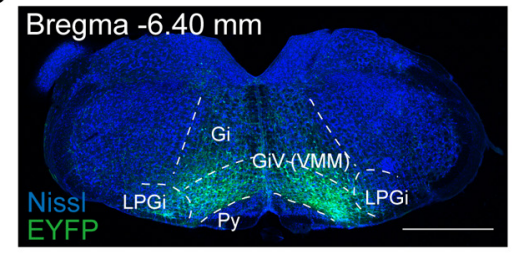

D
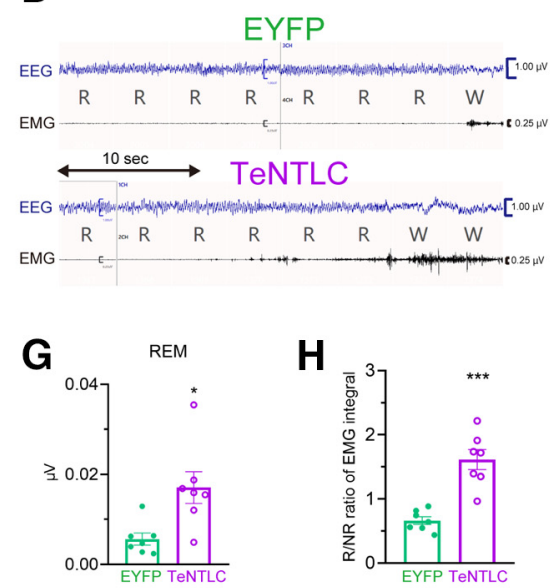

J
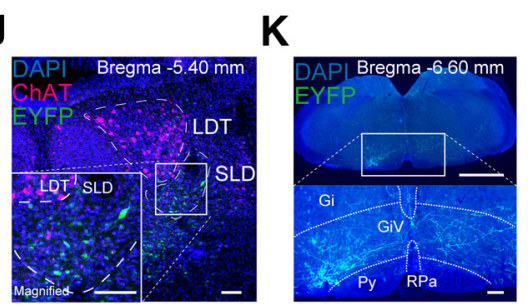

M
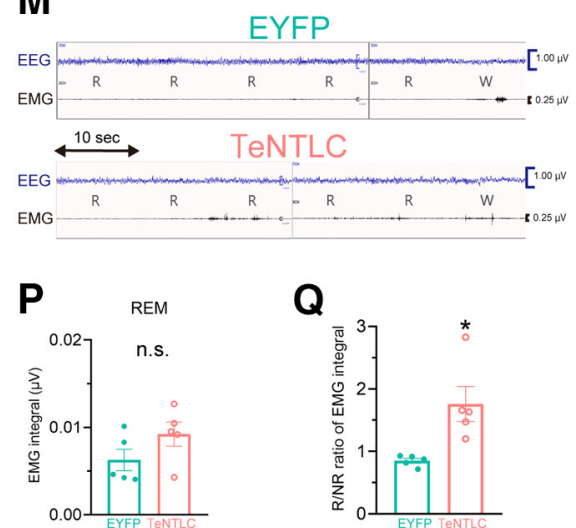

Figure 5. Silencing of Gly ${ }^{\mathrm{VMM}}$ neurons and Glu ${ }^{\mathrm{SLD} \rightarrow \mathrm{VMM}}$ pathways. $\boldsymbol{A}$, Schematic drawing of procedure. AAV2hSyn-FLEX(IoXP)-TeNTLC-P2A-EYFP or AAV10-EF1a-FLEX (IoXP)-EYFP was injected into the VMM of GlyT2-iCre mice. $\boldsymbol{B}$, Representative EYFP-positive neurons are shown. Scale bar: $1 \mathrm{~mm}$. $\boldsymbol{C}$, Schematic depicting EYFP expression areas in the brain; $n=7$ in each group. $\boldsymbol{D}$, Representative EEG trace (blue) and EMG trace (black) during REM sleep in control group (top) and TeNTLC group (bottom). $\boldsymbol{E}-\boldsymbol{G}$, EMG integral during wakefulness (E), NREM (F), and REM $(\boldsymbol{G}) . \boldsymbol{H}$, REM/NREM ratio of EMG integral. I, Schematics of AAV injection and representative image of expression. AAV-CAG-FLEX(Frt)-TeNTLC-P2A-EYFP or AAV-CAG-FLEX (Frt)-EYFP was injected into the SLD and CAV2-FLEX (IoXP)Flp was injected into the VMM of vGlut2-iresCre mice. $J$, Representative EYFP-positive neurons in SLD. ChAT is stained red whereas EYFP is stained green. Nissl is stained blue. Left lower shows a magnified image. Scale bar: $100 \mu \mathrm{m}$. $\boldsymbol{K}$, EYFP-positive axons in VMM. Scale bar: $1 \mathrm{~mm}$ (upper), $100 \mu \mathrm{m}$ (lower). $\boldsymbol{L}$, Outlines of EYFP expression sites in SLD; $n=5$ in each group. $\boldsymbol{M}$, Representative EEG trace (blue) and EMG trace (black) during REM sleep in control group (top) and TeNTLC group (bottom). $\boldsymbol{N}-\boldsymbol{P}$, EMG integral during wakefulness $(\boldsymbol{N})$, NREM ( 0 ), and REM $(\boldsymbol{P}) . \boldsymbol{Q}, \mathrm{REM} / \mathrm{NREM}$ ratio of EMG integral. All values are mean $\pm \mathrm{SEM} ; * p<0.05, * * p<0.01, * * * p<0.001$. Unpaired $t$ test. n.S., not significant.

reticular nucleus $(\mathrm{GiV})$, but not in the dorsal part of the gigantocellular reticular nucleus (Gi) and dorsal paragigantocellular nucleus (DPGi; Fig. 3C). We found TVA-mCherry-positive axons in Mo $5 \mathrm{~N}$, $7 \mathrm{~N}, 11 \mathrm{~N}, 12 \mathrm{~N}$, and the $\mathrm{AH}$ of the spinal cord. However, there were no mCherrypositive axons in $3 \mathrm{~N}, 4 \mathrm{~N}$, and $6 \mathrm{~N}$ (Fig. $3 D, E)$. These results indicate that Gly ${ }^{\mathrm{VMM} \rightarrow \mathrm{AH}}$ neurons send widespread collateral innervation to motoneurons except those that innervate extraocular muscles. Notably, we failed to find any TVA-mCherry projections to the $\mathrm{RN}$, vlPAG and monoaminergic nuclei, such as the LC and DR (Fig. 3D,E; Table 2). These observations suggest that axonal projections from Gly $\mathrm{BMM} \rightarrow \mathrm{AH}$ neurons are limited to motoneurons in the brainstem and spinal cord. Although we should be aware that the number of neurons infected with TVA-mCherry in the cTRIO experiments was smaller than that of mCherry+ cells in mice injected with AAV-mCherry into the VMM, we found a higher intensity of projections by Gly ${ }^{\mathrm{VMM} \rightarrow \mathrm{AH}}$ neurons to motor nuclei (Gly ${ }^{\mathrm{VMM}}, n=4$; $\mathrm{Gly}^{\mathrm{VMM} \rightarrow \mathrm{AH}}, n=4$; $t_{(3.915)}=3.179, p=0.0267$, unpaired $t$ test; Fig. 3F). So, it is not likely that the difference in projection regions stems from the numbers of starter cells in these two experiments. These observations suggest that there is a discrete population of Gly ${ }^{\mathrm{VMM}}$ neurons among Gly ${ }^{\mathrm{VMM}}$ neurons that are specialized to regulate motoneurons (Gly ${ }^{\mathrm{VMM}} \rightarrow \mathrm{Mn}$ neurons).

\section{Input of motor neuron-projecting} glycinergic neurons in VMM

In the cTRIO experiment, we found many GFP-single-positive neurons (input neurons) in the brainstem, such as the deep mesencephalic nucleus (DpMe), PAG, superior colliculus (SC), SLD and pontine reticular nucleus (Pn; Fig. $4 A, G$ ). Substantial numbers of input neurons were found in the SLD and laterodorsal tegmental nucleus (LDT), which are in close proximity to each other, and have been implicated in REM sleep regulation (Scammell et al., 2017). To examine whether GFP-labeled input neurons in this region are cholinergic or glutamatergic, we combined IHC and FISH. We found that GFP-labeled SLD neurons were co-localized with Vglut2 but not with ChAT. IHC studies revealed that all GFP labeled neurons in the SLD (11/11, n=3) were negative for ChAT and FISH experiments showed that $85.7 \%$ of GFP labeled neurons in the $\operatorname{SLD}(6 / 7, n=3)$ were positive for Vglut2 but not ChAT (Fig. 4B,C). This suggests that glutamatergic neurons in the SLD (Glu ${ }^{\text {SLD }}$ neurons), but 
not cholinergic neurons in the LDT make direct synaptic contact with Gly ${ }^{\mathrm{VMM}} \rightarrow$ Mn neurons.

As a control, we also performed simple trans-synaptic retrograde tracing of Gly ${ }^{\mathrm{VMM}}$ neurons in GlyT2-Cre mice to examine whether Gly ${ }^{\mathrm{VMM}} \rightarrow \mathrm{Mn}$ neurons have different input from Gly ${ }^{\mathrm{VMM}}$ neurons. We injected Cre-activatable AAV vectors, AAV10-CAG-FLEX(LoxP)-TVA-mCherry and AAV10-CAG-FLEX (LoxP)-RG, into the left VMM to express TVA and RG in Gly ${ }^{\mathrm{VMM}}$ neurons. Fourteen days later, we injected $S A D \triangle G-G F P(E n v A)$ into the left VMM (Fig. 4D). Starter neurons were expressed in the VMM (Fig. 4E). We counted the input neurons in each brain region and compared the percentage of input neurons in each region between the cTRIO study ( $\mathrm{Gly}^{\mathrm{VMM} \rightarrow \mathrm{AH}}$ ) and conventional tracing $\left(\mathrm{Gly}^{\mathrm{VMM}}\right)$ study. Ma ny brainstem regions, such as the DpMe, PAG, SC, SLD, and Pn, showed moderate numbers of input cells with a similar proportional distribution in both Gly ${ }^{\mathrm{VMM} \rightarrow \mathrm{AH}}$ and $\mathrm{Gly}^{\mathrm{VMM}}$ cells (Fig. $4 F-G$ ). However, Gly ${ }^{\mathrm{VMM} \rightarrow \mathrm{AH}}$ neurons receive a larger number of input neurons from local $\mathrm{Gi}$ and $\mathrm{GiA} / \mathrm{GiV}$ neurons than from Gly ${ }^{\mathrm{VMM}}$ neurons, whereas Gly ${ }^{\mathrm{VMM}}$ receive more input from neurons in the vestibular nucleus and cerebellum than from Gly ${ }^{\mathrm{VMM} \rightarrow \mathrm{AH}}$, suggesting that Gly ${ }^{\mathrm{VMM}} \rightarrow \mathrm{Mn}$ neurons have an input pattern that is different from that of other Gly $^{\text {VMM }}$ neurons (Fig. 4G).

\section{Silencing the SLD $\rightarrow$ VMM pathway resulted in REM sleep without atonia}

To examine the role of Gly ${ }^{\mathrm{VMM}}$ neurons in the regulation of REMatonia, we used tetanus toxin light chain (TeTNLC), which blocks SNARE-mediated neurotransmission, by injecting AAV-FLEX (loxP)-TeNTLC-EYFP stereotaxically into the VMM of GlyT2iCre mice (Fig. 5A). We confirmed that EYFP-positive cells were exclusively found in the VMM (Fig. 5B,C). As a control, we used GlyT2-iCre mice with injection of $A A V$-FLEX(loxP)EYFP into the VMM. Two weeks after the surgery, we recorded EEG/EMG to analyze sleep/wakefulness states in these mice. In control mice $(n=7)$, REM sleep was typically characterized by high theta-frequency EEG activity and silent EMG (Movie 1; Fig. 5D). In contrast, all TeNTLC-expressing mice $(n=7)$ exhibited abnormalities during REM sleep, characterized by excessive body and limb movements despite clear REM-like theta frequency EEG (Movie 2; Fig. 5D). We quantified EMG integral values in each stage in each mouse and found that TeNTLC-expressing mice showed higher EMG integral value during REM sleep than control mice (EYFP, $n=7$; TeNTLC, $n=7 ; t_{(7.736)}=3.043, p=0.0292$, unpaired $t$ test; Fig. $5 G$ ). EMG integral value during wakefulness and NREM did not show differences between genotypes (wakefulness: $t_{(12)}=0.9134$, $p=0.3790$; NREM: $t_{(12)}=0.989, p=0.3422$, unpaired $t$ test; Fig. $5 E, F)$. REM/NREM ratio of EMG integral ratio in TeNTLCexpressing mice was higher than that in control mice $\left(t_{(7.79)}=\right.$ 5.689, $p=0.0005$, unpaired $t$ test; Fig. $5 H$ ). These results indicate that function of Gly ${ }^{\mathrm{VMM}}$ neurons is indispensable for inducing REM-atonia.

In the cTRIO tracing study, we identified input neurons of Gly ${ }^{\mathrm{VMM}} \rightarrow \mathrm{Mn}$ neurons in several brain stem regions (Fig. $4 A-C, G)$. Among them, we focused on the SLD, which has been implicated in the regulation of REM sleep (Boissard et al., 2002; Clément et al., 2011; Sakai, 2015). To examine the role of glutamatergic neurons in the SLD that send projections to the VMM (Glu ${ }^{\mathrm{SLD} \rightarrow \mathrm{VMM}}$ neurons), we injected $C A V 2-F L E X(\operatorname{lox} P)-F l p$ into the VMM and $A A V$-FLEX(Frt)-

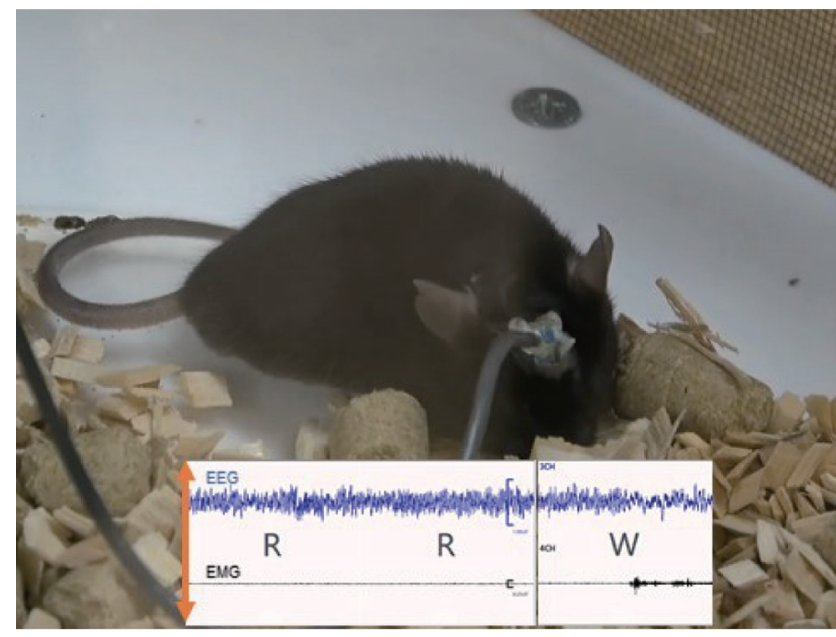

Movie 1. Control mice (EYFP-expressing GlyT2-iCre mice) during REM sleep. [View online]

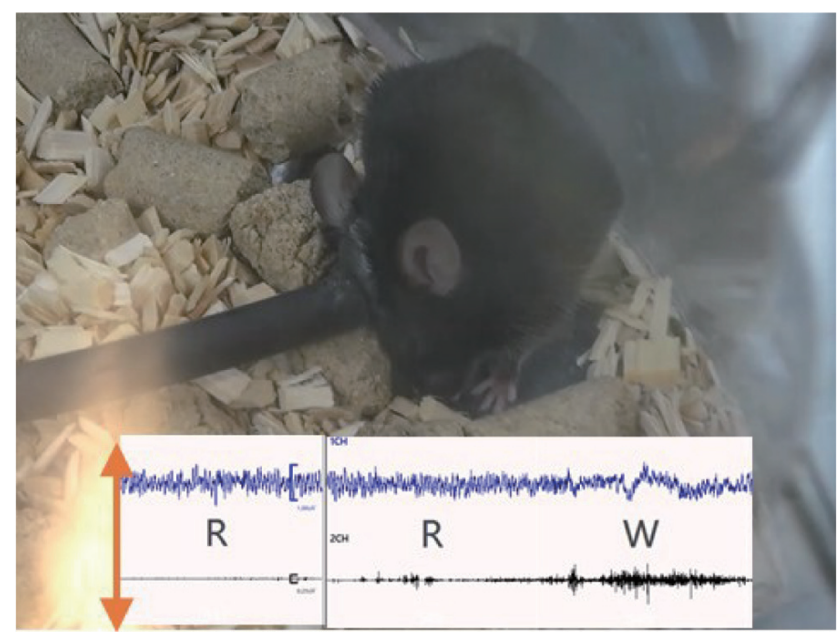

Movie 2. TeNTLC-expressing GlyT2-iCre mice during REM sleep. [View online]

TeNTLC-EYFP (or AAV-FLEX(Frt)-EYFP as control) into the SLD of $v$ Glut2-iresCre mice (Fig. 5I). We confirmed that expression of EYFP-positive cells was mainly located in the SLD (Fig. 5J,L), and EYFP-positive axons were found in the VMM (Fig. $5 K)$, which showed a similar distribution to that of starter neurons in the cTRIO experiment (Fig. 3C). Two weeks after the surgery, we observed no significant difference in EMG integral value in each stage (EYFP, $n=5$; TeNTLC, $n=5$; wakefulness: $t_{(8)}=$ 0.8371, $p=0.4268$; NREM: $t_{(8)}=0.9457, p=0.3720$; REM: $t_{(8)}=1.599, p=0.1484$, unpaired $t$ test; Fig. $\left.5 N-P\right)$. However, $\mathrm{R} / \mathrm{NR}$ ratio of EMG integral was significantly higher in TeNTLC-injected mice than in control mice $\left(t_{(4.153)}=3.219\right.$, $p=0.0306$, unpaired $t$ test; Fig. 5Q). These results indicate that $\mathrm{Glu}^{\mathrm{SLD} \rightarrow \mathrm{VMM}}$ neurons play an important role in evoking muscle atonia during REM sleep.

\section{Silencing the SLD $\rightarrow$ VMM pathway blocked cataplectic attacks in narcolepsy mice}

Next, we examined whether the same pathway is also involved in exhibiting cataplexy in narcolepsy model mice by manipulating Gly ${ }^{\mathrm{VMM}}$ neurons and Glu ${ }^{\mathrm{SLD} \rightarrow \mathrm{VMM}}$ neurons. We crossed GlyT2-iCre mice with orexin-ataxin3 mice (Hara et al., 
A

$A A V-F L E X($ loxP)-EYFP or AAV-FLEX(IoXP)-TeNTLC-EYFP

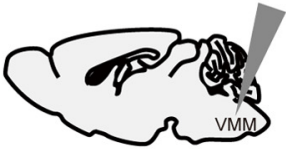

GlyT2-iCre;orexin-ataxin3
B

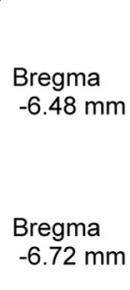

EYFP

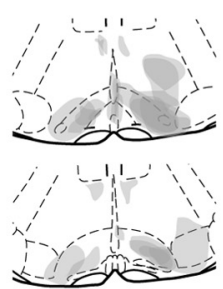

TeNTLC

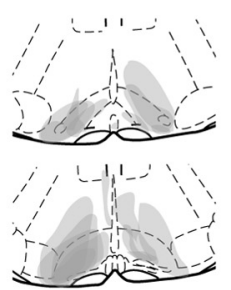

C

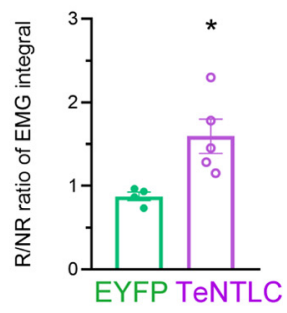

D

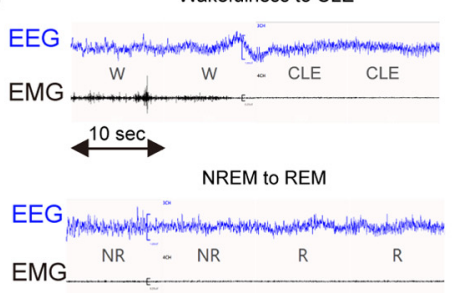

CLE to Wakefulness

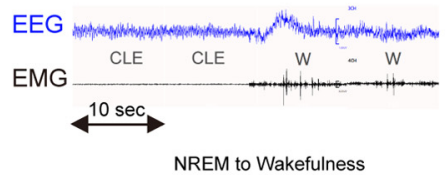

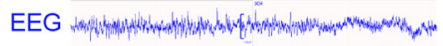
EMG NR NR $\quad W \quad W$

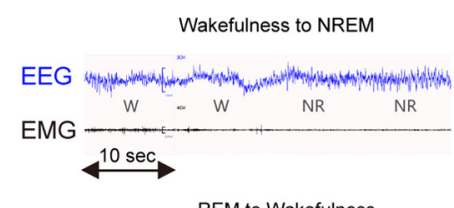

REM to Wakefulness

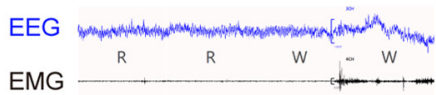

\section{CLEs}
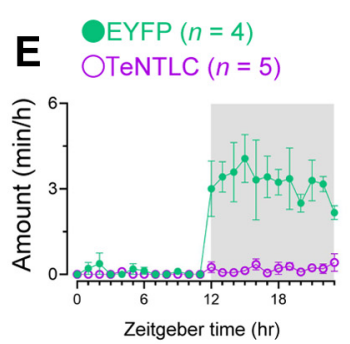

I

AAV-FLEX(Frt)-EYFP or AAV-FLEX(Frt)-TeNTLC-EYFP

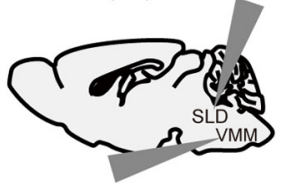

CAV2-FLEX(loxP)-FIp

vGlut2-iresCre;orexin-ataxin3

\section{$\mathbf{F}$}

Mice were fed chocolate during dark phase.

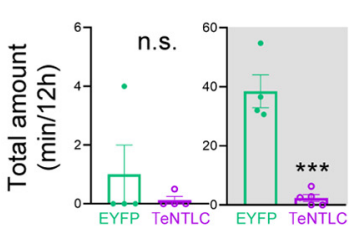

G

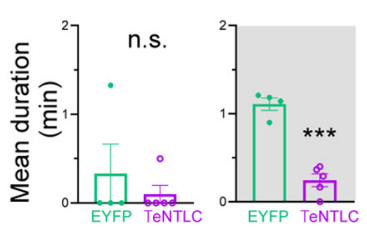

$\mathbf{J}$

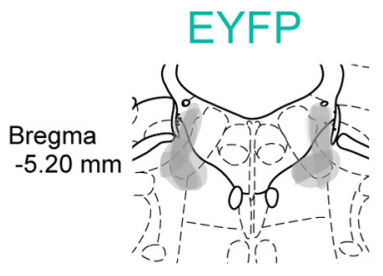

CLES

\section{H}

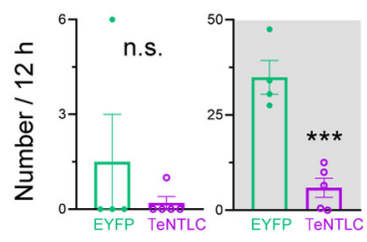

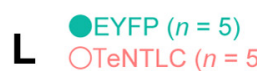

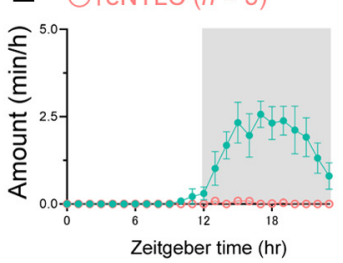

Mice were fed chocolate during dark phase.

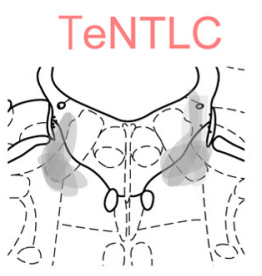

K

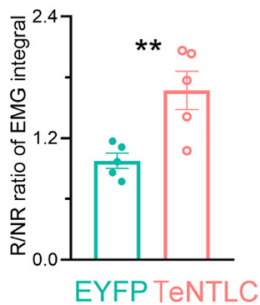

M

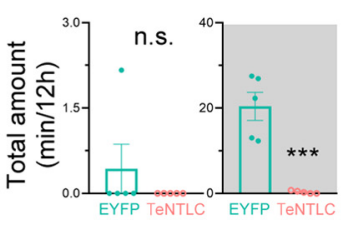

$\mathbf{N}$

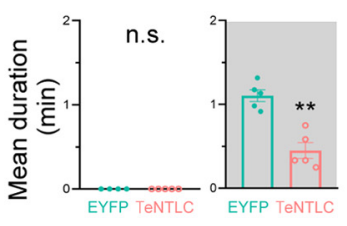

0

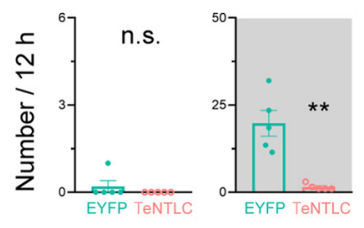

Figure 6. Silencing of Gly ${ }^{\mathrm{VMM}}$ neurons and Glu ${ }^{\text {SLD } \rightarrow \mathrm{VMM}}$ pathway in narcolepsy model mice. A, Experimental procedure. AAV2-hSyn-FLEX(loxP)-TeNTLC-P2A-EYFP or AAV10-EF1a-FLEX (loxP)-EYFP was injected into the VMM of GlyT2-iCre;orexin-ataxin3 mice. $\boldsymbol{B}$, Area of EYFP expression site in VMM. EYFP, $n=4$; TeNTLC, $n=5$. C, REM/NREM ratio of EMG integral. $\boldsymbol{D}$, Representative EEG traces (blue) and EMG traces (black) of transitions from wakefulness to CLE (top left), from CLE to wakefulness (top middle), from wakefulness to NREM (top right), from NREM to REM (bottom left), from NREM to wakefulness (bottom middle), and from REM to wakefulness (bottom right). $\boldsymbol{E}$, Time of (LEs in a day. $\boldsymbol{F}$, Total amount of (LEs. $\boldsymbol{G}$, Mean episode duration of CLEs. $\boldsymbol{H}$, Episode number of (LEEs. $\boldsymbol{I}$, Experimental procedure. AAV2CAG-FLEX(Frt)-TeNTLC-P2A-EYFP or AAV10-CAG-FLEX(Frt)-EYFP was injected into the SLD and CAV2-FLEX(loXP)-Flp was injected into the VMM of VGlut2-ires(re:orexin-ataxin3 mice. J, Areas containing EYFP expression in SLD; $n=5$ in each group. $\boldsymbol{K}$, REM/NREM ratio of EMG integral. $\boldsymbol{L}$, Time of CLEs in a day. $\boldsymbol{M}$, Total amount of CLEs. $\boldsymbol{N}$, Mean episode duration of CLEs. $\mathbf{0}$, Episode number of CLEs. All values are mean \pm $\mathrm{SEM} ; * p<0.05, * * p<0.01, * * * p<0.001$. Unpaired $t$ test for single variables and two-way ANOVA followed by Bonferroni post hoc tests for multiple variables. n.S., not significant.

2001) to obtain narcoleptic mice with expression of Cre in glycine neurons (GlyT2-iCre mice;orexin-ataxin3). We first injected $A A V$ FLEX (loxP)-TeNTLC-EYFP or AAV-FLEX (loxP)-EYFP as control into the VMM of GlyT2-iCre;orexin-ataxin3 mice (Fig. 6A). Two weeks after the surgery, mice were subjected to sleep analysis. After the experiments, we confirmed that EYFP-positive cells were localized in the VMM (Fig. 6B). EMG integral R/NR ratio was higher in TeNTLC-expressing mice than in control mice (EYFP, 
$n=4$; TeNTLC, $n=5 ; t_{(4.401)}=2.785, p=0.0446$, unpaired $t$ test; Fig. $6 C$ ).

We gave mice a chocolate at the onset of the dark period, because it was shown to increase the bout number of cataplexy (Oishi et al., 2013). According to previous studies, we defined the sudden onset of REM sleep from wakefulness as the criteria for CLEs (Scammell et al., 2009; Hasegawa et al., 2014, 2017; Fig. 6D). We found that TeNTLC-expressing mice exhibited a lower amount of CLEs $\left(F_{(1,7)}=61.30, p=0.0001\right.$, ANOVA; Fig. 6E). Total amount of CLEs during the dark period was decreased by $92 \%$ in TeNTLC-expressing mice (dark period: $t_{(3.261)}=$ 6.362, $p=0.0061$, unpaired $t$ test; Fig. $6 F$ ). The mean duration of CLEs was significantly shortened by $78 \%$ (dark period: $t_{(7)}=8.325$, $p<0.0001$, unpaired $t$ test; Fig. $6 G$ ), and the episode number was significantly decreased by $83 \%$ in TeNTLC-expressing mice (dark period: $t_{(7)}=$ 6.037, $p=0.0005$, unpaired $t$ test; Fig. $6 H$ ), showing that Gly ${ }^{\mathrm{VMM}}$ neurons play an important role in evoking cataplexy.

Although our observations suggested that Gly ${ }^{\mathrm{VMM}}$ neurons are common brain stem output neurons that induce atonia during both REM-atonia and cataplexy, there is a possibility that the systems that activate these cells might be different from those that induce REM atonia, because we found that Gly ${ }^{\mathrm{VMM}} \rightarrow \mathrm{Mn}$ neurons have multiple input regions (Fig. $4 A-C, G$ ).

We examined the effect of pathway-selective silencing of Glu ${ }^{\mathrm{SLD} \rightarrow \mathrm{VMM}}$ neurons on cataplexy. We crossed vGlut2-iresCre mice with orexin-ataxin 3 mice to obtain vGlut2-iresCre; orexin-ataxin 3 mice, and injected CAV2-FLEX (loxP)-Flp into the VMM and $A A V-F L E X(F r t)-$ TeNTLC-EYFP or AAV-FLEX(Frt)-EYFP as control into the SLD (Fig. 6I). We confirmed that EYFP-positive neurons were localized in the SLD of these mice after the experiments (Fig. 6J). Two weeks after the surgery, we analyzed the sleep/wake patterns of these mice. We observed that the EMG integral $\mathrm{R} /$ $\mathrm{NR}$ ratio was significantly higher in the TeNTLC-injected group than in control mice (EYFP, $n=5$; TeNTLC, $n=5 ; t_{(8)}=3.415$, $p=0.0092$, unpaired $t$ test; Fig. $6 K)$.

Under chocolate feeding, we found that the TeNTLC group showed a much smaller amount of CLEs throughout the day $\left(F_{(1,8)}=\right.$ 43.52, $p=0.0002$, ANOVA, Fig. $6 L)$. ANOVA; Fig. $6 L$ ). Total amount, mean duration, and episode number of CLEs during the dark period were significantly decreased in the TeNTLC-injected group (total amount during light period: $t_{(4)}=1.000, p=0.3739$; total amount during dark period: $t_{(4.016)}=6.109$, $p=0.0036$; mean duration during light period: $t_{(4)}=1.000$, $p=0.3739$; mean duration during dark period: $t_{(8)}=5.578, p=$ 0.0005 ; episode number during light period: $t_{(8)}=1.000, p=0.3739$;

\section{AAV-FLEX(loxP)-EYFP or $A A V-F L E X($ loxP $)-T e N T L C-E Y F P$}

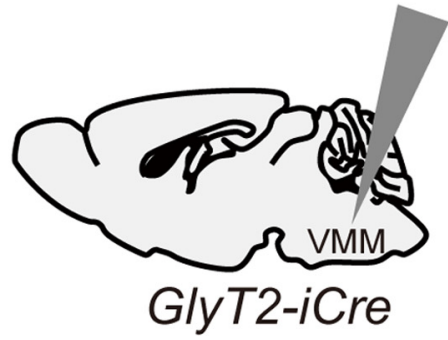

OEYFP $(n=7)$ OTeNTLC $(n=7)$
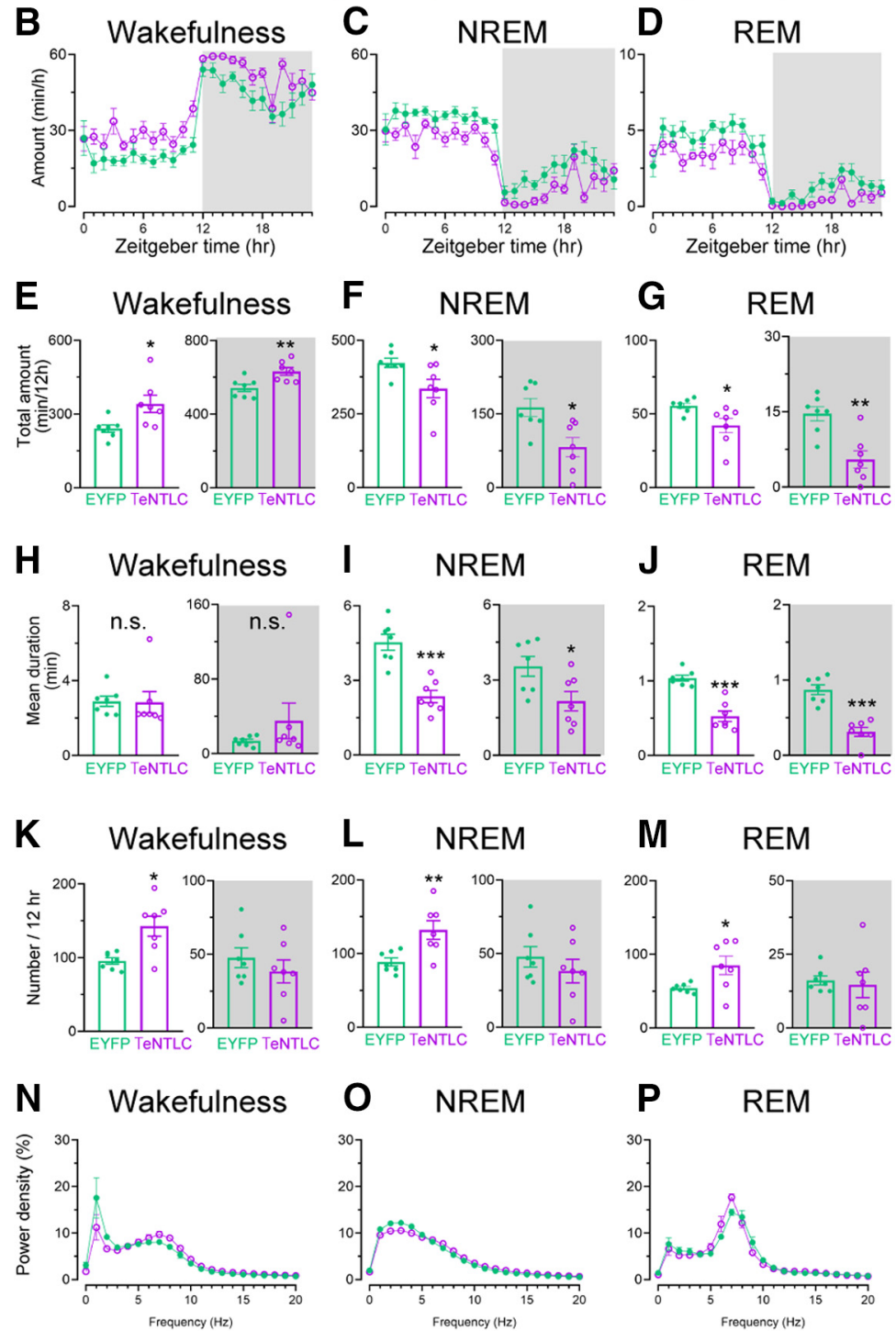

Figure 7. Silencing of Gly ${ }^{\mathrm{VMM}}$ neurons affects sleep/wake architecture. $\boldsymbol{A}$, Experimental procedure. $\boldsymbol{B}-\boldsymbol{D}$, Time in each state. Wakefulness $(\boldsymbol{B})$, NREM $(\boldsymbol{C})$ and REM $(\boldsymbol{D} ; n=7$ in each group). $\boldsymbol{E}-\boldsymbol{G}$, Total amount of wakefulness $(\boldsymbol{E})$, $\operatorname{NREM}(\boldsymbol{F})$, and REM $(\boldsymbol{G}) . \boldsymbol{H}-\boldsymbol{J}$, Mean episode duration of wakefulness $(\boldsymbol{H})$, NREM $(\boldsymbol{I})$, and REM $(\boldsymbol{J}) . \boldsymbol{K}-\boldsymbol{M}$, Episode number of wakefulness $(\boldsymbol{K})$, NREM $(\boldsymbol{L})$, and REM $(\boldsymbol{M})$. $\boldsymbol{N}-\boldsymbol{P}$, EEG power density during wakefulness $(\boldsymbol{M})$, NREM $(\boldsymbol{O})$, and REM (P). All values are mean \pm SEM; $* p<0.05, * * p<0.01, * * * p<0.001$. Unpaired $t$ test for single variables and two-way ANOVA followed by Bonferroni post hoc tests for multiple variables. n.s., not significant.

episode number during dark period: $t_{(4.088)}=4.928, p=0.0075$; Fig. $6 M-O)$. These results suggest that glutamatergic input to Gly ${ }^{\mathrm{VMM}} \rightarrow \mathrm{Mn}$ neurons also plays an important role in generating cataplexy in narcoleptic mice. 
Table 3. Total time spent in each state (min), episode duration (s), and episode number during light phase and dark phase

\begin{tabular}{|c|c|c|c|c|c|c|}
\hline & \multicolumn{2}{|l|}{ Wakefulness } & \multicolumn{2}{|l|}{ NREM } & \multicolumn{2}{|l|}{ REM } \\
\hline & EYFP & TeNTLC & EYFP & TeNTLC & EYFP & TeNTLC \\
\hline \multicolumn{7}{|l|}{ Light period } \\
\hline Total time (min) & $212.4 \pm 20.1$ & $241.6 \pm 31.7$ & $438.9 \pm 18.8$ & $441.2 \pm 27.5$ & $68.7 \pm 2.5$ & $37.1 \pm 6.1 * *$ \\
\hline Episode duration (s) & $124.8 \pm 17.4$ & $110.2 \pm 12.3$ & $236.5 \pm 5.2$ & $196.6 \pm 17.3$ & $64.6 \pm 1.7$ & $30.3 \pm 3.6 * * *$ \\
\hline Episode number & $105.7 \pm 5.2$ & $129.8 \pm 5.9$ & $111.7 \pm 4.9$ & $136.8 \pm 4.6$ & $63.9 \pm 2.3$ & $72.0 \pm 6.4$ \\
\hline \multicolumn{7}{|l|}{ Dark period } \\
\hline Total time (min) & $552.0 \pm 15.2$ & $566.7 \pm 64.0$ & $153.2 \pm 14.5$ & $145.7 \pm 60.1$ & $14.9 \pm 1.5$ & $7.6 \pm 4.0$ \\
\hline Episode duration (s) & $1112.4 \pm 232.9$ & $2062.8 \pm 673.3$ & $247.8 \pm 19.9$ & $178.4 \pm 16.0$ & $50.1 \pm 2.2$ & $23.2 \pm 4.6 *$ \\
\hline Episode number & $37.3 \pm 5.2$ & $48.7 \pm 19.0$ & $38.4 \pm 5.2$ & $48.7 \pm 19.3$ & $17.5 \pm 1.1$ & $14.5 \pm 6.0$ \\
\hline
\end{tabular}

All values are mean \pm SEM; $* p<0.05, * * p<0.01, * * * p<0.001$. Unpaired $t$ test. EYFP, $n=5$; TeNTLC, $n=5$.

\section{Role of Gly ${ }^{\mathrm{VMM}}$ neurons in regulation of sleep/wakefulness states}

While analyzing sleep/wakefulness characteristics of mice with expression of TeNTLC in Gly ${ }^{\mathrm{VMM}}$ neurons (Fig. 7A), we found that these mice showed longer wakefulness time (EYFP, $n=7$; TeNTLC, $n=7 ; F_{(1,12)}=9.703, p=0.0089$, ANOVA; Fig. $\left.7 B\right)$, along with decreased NREM and REM sleep times as compared with controls (NREM: $F_{(1,12)}=8.786, p=0.0018$; REM: $F_{(1,12)}=$ $12.20, p=0.0044$, ANOVA; Fig. $7 C, D)$. Total amount of wakefulness was significantly longer in TeNTLC-expressing mice (light period: $t_{(12)}=2.645, p=0.0214$; dark period: $t_{(12)}=3.158$, $p=0.0082$, unpaired $t$ test; Fig. $7 E$ ), and total amounts of both NREM and REM were significantly shorter than those in control (light period NREM: $t_{(12)}=2.511, p=0.0273$; dark period NREM; $t_{(12)}=3.042, p=0.0102$, unpaired $t$ test; light period REM: $t_{(7.6)}=2.572, p=0.0344$; dark period REM: $t_{(12)}=4.082$, $p=0.0015$, unpaired $t$ test; Fig. $7 F, G)$. The mean duration of wakefulness was not changed (light period: $t_{(12)}=0.08698$, $p=0.9321$; dark period: $t_{(12)}=1.117, p=0.2861$, unpaired $t$ test; Fig. $7 H$ ). Episode number of wakefulness was higher during the light period in TeNTLC-expressing mice, but was not different during the dark period compared with control (light period; $t_{(12)}=3.151, p=0.0084$; dark period; $t_{(12)}=0.9027, p=0.3844$, unpaired $t$ test; unpaired $t$ test; Fig. $7 K)$. Mean durations of both NREM and REM were significantly shorter in TeNTLC-expressing mice (light period NREM: $t_{(12)}=5.432, p=0.0002$; dark period NREM: $t_{(12)}=2.527, p=0.0266$; light period REM: $t_{(12)}=$ 6.416, $p<0.0001$; dark period REM: $t_{(12)}=6.361, p<0.0001$, unpaired $t$ test; Fig. $7 I, J)$, whereas the episode number was higher in the TeNTLC group as compared with control during the light period, but was not different during the dark period (light period NREM: $t_{(7.198)}=3.288, p=0.0128$; dark period NREM; $t_{(12)}=$ $0.9070, p=0.3823$; light period REM: $t_{(6.395)}=2.450, p=0.0473$, Welch's $t$ test; dark period REM: $t_{(7.502)}=0.3268, p=0.7527$, unpaired $t$ test; Fig. $7 L, M)$. EEG power densities in each stage were comparable between groups (wakefulness: $F_{(1,12)}=2.106$, $p=0.1723$; NREM: $F_{(1,12)}=0.1307, p=0.7240$; REM: $F_{(1,12)}=$ $0.6863, p=0.4236$, ANOVA; Fig. $7 N-P$ ). These results suggest that Gly ${ }^{\mathrm{VMM}}$ neurons also play a role in promoting and consolidating both NREM and REM sleep.

Next, we examined the effect of inhibition of Glu ${ }^{\mathrm{SLD} \rightarrow \mathrm{VMM}}$ neurons to determine to what extent the effect of silencing glycine neurons in the VMM on NREM and REM sleep is driven by the subset of glycine neurons receiving input from the SLD. We injected CAV2-FLEX(loxP)-Flp into the VMM and AAV-FLEX (Frt)-TeNTLC-EYFP (AAV-FLEX(Frt)-EYFP as control) into the SLD in $v$ Glut2-iresCre mice. Two weeks after the surgery, we analyzed sleep/wake patterns of these mice. We confirmed that
EYFP-positive cells were exclusively found in the SLD. Two weeks after the surgery, we recorded EEG/EMG to analyze sleep/ wakefulness states in these mice. We did not find a significant difference in wakefulness and NREM between the experimental and control groups, suggesting that the observed wake-promoting and NREM-suppressing effects of silencing Gly ${ }^{\mathrm{VMM}}$ neurons are not likely solely driven by SLD input (EYFP, $n=5$; TeNTLC, $n=5$; Table 3 ). However, the total amount of REM sleep during the light period and the episode duration during both periods were significantly decreased in the TeNTLC-expressing group (light period REM, $t_{(8)}=4.304, p=0.0026$; episode duration light period REM, $t_{(8)}=7.641, p<0.0001$; episode duration dark period REM, $\left.t_{(8)}=4.704, p=0.0015\right)$. These results suggest that the REM-suppressing effect of silencing Gly ${ }^{\mathrm{VMM}}$ neurons is mediated by the Glu ${ }^{\mathrm{SLD}} \rightarrow \mathrm{Gly}^{\mathrm{VMM}}$ pathway.

\section{Discussion}

REM sleep is characterized by a desynchronized EEG and paralysis of anti-gravity skeletal muscles. Several studies have suggested that Gly ${ }^{\mathrm{VMM}}$ neurons are involved in the induction of REM-atonia. For example, a recent study showed that silencing of $\mathrm{Vgat}$ mRNA in the VMM by RNA-interference induced REM sleep without atonia in rats (Garcia et al., 2018). However, the precise input and output architecture of REM-atonia-inducing Gly ${ }^{\mathrm{VMM}}$ neurons has been unknown.

In this study, we first identified a subpopulation of Gly ${ }^{\mathrm{VMM}}$ neurons that sends innervations exclusively to somatic motoneurons (Gly ${ }^{\mathrm{VMM}} \rightarrow \mathrm{Mn}$ neurons). Although Gly ${ }^{\mathrm{VMM}} \rightarrow \mathrm{Mn}$ neurons send projections to brainstem motoneurons and all levels of motoneurons in the $\mathrm{AH}$ of the spinal cord, these neurons do not send projections to other brain stem regions including oculomotor neurons (Fig. 3D). This suggests that Gly ${ }^{\mathrm{VMM}} \rightarrow \mathrm{Mn}$ neurons send widespread collaterals to all motoneurons but these projections are limited to somatomotoneurons. Since anterograde tracing studies of whole Gly ${ }^{\mathrm{VMM}}$ neuron populations suggest that these neurons send axonal projections to other brain regions including the $\mathrm{RN}, \mathrm{DpMe}$, vlPAG, and monoaminergic nuclei, such as the LC and the DR (Table 2; Fig. $3 E$ ), this study showed that Gly ${ }^{\mathrm{VMM}} \rightarrow \mathrm{Mn}$ neurons constitute a unique subpopulation among Gly ${ }^{\mathrm{VMM}}$ neurons with particular input and output architectures. Recent findings suggested that neurons in the dorsal medial medulla (DMM) send projections to the $3 \mathrm{~N}, 4 \mathrm{~N}$, and $6 \mathrm{~N}$, and contribute to REM during REM sleep (Gutierrez Herrera et al., 2019). Taken together, the medulla might have two distinct roles in controlling muscle activities during REM sleep; the DMM contributes to eye movements by activating $3 \mathrm{~N}, 4 \mathrm{~N}$, and $6 \mathrm{~N}$, whereas the VMM contributes to muscle atonia by inhibiting all somatic motoneurons except $3 \mathrm{~N}, 4 \mathrm{~N}$, and $6 \mathrm{~N}$. 
Many studies have suggested that SLD neurons play a pivotal role in the maintenance of REM sleep, because SLD neurons are specifically activated during REM sleep (Boissard et al., 2002; Lu et al., 2006; Clément et al., 2011; Sakai, 2015). Our rabies-mediated retrograde study of Gly ${ }^{\mathrm{VMM}} \rightarrow \mathrm{Mn}$ revealed that glutamatergic neurons in the SLD make direct synaptic contacts with these neurons (Fig. 4C). Moreover, silencing the $\mathrm{Glu}{ }^{\mathrm{SLD} \rightarrow \mathrm{VMM}}$ pathway by specific expression of TeNTLC suppressed muscle atonia during REM sleep (Fig. 5M,Q). These observations are consistent with a previous report that silencing of Slc32a1 mRNA, which encodes a vesicular transporter of inhibitory amino acids, in the ventral part of the gigiantocellular nucleus (GiV; one region of VMM; Garcia et al., 2018) or silencing of Slc17a6 mRNA, which encodes Vglut2, in the SLD impaired REM-atonia (Valencia Garcia et al., 2017).

We confirmed that silencing of Gly ${ }^{\mathrm{VMM}}$ neurons by expressing TeNTLC impaired REM-atonia (Fig. 5D,H). Moreover, we found that CLEs were markedly suppressed in narcolepsy model mice (orexin-ataxin3) when TeNTLC was expressed in Gly ${ }^{\mathrm{VMM}}$ neurons or Glu ${ }^{\mathrm{SLD} \rightarrow \mathrm{VMM}}$ neurons (Fig. $6 E-H, L-O)$. A previous in vivo electrophysiological study in dogs demonstrated that a subset of neurons in the medial medulla fire at high rates during both REM sleep and cataplexy (Siegel et al., 1991). Our results suggest that these activations generate muscle atonia during both REM and cataplexy.

Our silencing study also showed that Glu ${ }^{\text {SLD }}$ neurons play an important role in muscle atonia during both REM sleep and CLEs. Consistently, a recent finding suggested that chemogenetic activation of glutamatergic neurons in the SLD induces cataplexy-like states even in wild-type mice, although they found that chemogenetic inactivation of glutamatergic neurons in the SLD failed to prevent CLEs in orexin knockout mice (Torontali et al., 2019). This difference might stem from the method used in each study. Although CNO application is shown to hyperpolarize the membrane potential of hM4Di-expressing neurons, the effect of TeNTLC, which blocks vesicular transmission, might be more effective than that of a chemogenetic approach (Schiavo et al., 1992).

We also found that mice with expression of TeNTLC in Gly ${ }^{\mathrm{VMM}}$ neurons showed an increase in wakefulness along with decreases in NREM and REM sleep (Fig. 7). An anterograde tracing study suggested that some populations of Gly ${ }^{\mathrm{VMM}}$ neurons, but not Gly ${ }^{\mathrm{VMM}} \rightarrow \mathrm{Mn}$ neurons, innervate monoaminergic nuclei such as the LC and DR, which are known to be implicated in maintenance of wakefulness (Figs. 2C, 3E; Table 2). These projections might play a role in promoting sleep. Consistently, a recent study showed that optogenetic activation of GAD2-positive axon terminals in the LC and DR arising from the VMM promotes NREM sleep (Zhong et al., 2019). However, they found that optogenetic inactivation of GAD2-positive neurons in the VMM does not affect EMG activity during REM sleep, which is not consistent with our data. This discrepancy might stem from a difference in genetic labeling since the prior work inactivated Gad2-expressing neurons, but not GlyT2-expressing neurons. Whereas most GABA and glycine neurons are co-localized in the VMM, some populations of GABA neurons and glycinergic neurons are distributed separately in the medulla (Tanaka et al., 2003; Tanaka and Ezure, 2004). These results underscore the importance of glycinergic neurotransmission in REM-atonia. Furthermore, GABA-cotransmitting and glycine-cotransmitting neurons in some brainstem regions during late embryonic development are known to differentiate into GABAergic and glycinergic neurons in the later developmental phase (Nabekura et al.,
2004; Hirrlinger et al., 2019), although these studies may not apply to the VMM.vlPAG-projecting GABA/glycinergic neurons in the VMM were shown to promote REM sleep (Weber et al., 2015). We found glycinergic fibers in the vlPAG arising from the VMM (Figs. 2C, 3E; Table 2), suggesting that the TeNTLC might affect this REM-promoting population, resulting in a decrease in REM sleep time.

Our study suggested that $\mathrm{Glu}^{\mathrm{SLD} \rightarrow \mathrm{VMM}}$ neurons excited Gly ${ }^{\mathrm{VMM}} \rightarrow \mathrm{Mn}$ neurons to induce cataplexy. Since narcolepsy is caused by a loss of orexin neurons, Glu ${ }^{\mathrm{SLD} \rightarrow \mathrm{VMM}}$ neurons should be under the influence of the orexin system. Also, since cataplexy is triggered by strong emotions, this pathway should receive influences from the systems that regulate emotion. Our previous study showed that orexin neurons inhibit cataplexy through activation of DR serotonin neurons by reducing amygdala activity (Hasegawa et al., 2017). These findings suggest that amygdala neurons might affect the activity of Glu ${ }^{\text {SLD } \rightarrow \text { VMM }}$ neurons (Mahoney et al., 2017). Another candidate mechanism by which $\mathrm{Glu}^{\mathrm{SLD}}$ is regulated downstream of orexinergic neurons involves the ventrolateral PAG (vlPAG). A recent study demonstrated that optogenetic activation of GABAergic axons in the dorsal pons originating from the vlPAG suppressed REM sleep (Weber et al., 2018). This observation suggests the possibility that orexin neurons activate GABAergic neurons in the vlPAG that suppress REMatonia by inhibiting $\mathrm{Glu}^{\mathrm{SLD} \rightarrow \mathrm{VMM}}$ neurons, because GABAergic neurons in the vlPAG receive dense innervations from orexin neurons (Peyron et al., 1998; Sakurai, 2014) and from GABAergic neurons in the central amygdala (CeA; Rizvi et al., 1991; Oka et al., 2008; Tovote et al., 2016; Han et al., 2017). Orexin neurons might inhibit $\mathrm{Glu}^{\mathrm{SLD} \rightarrow \mathrm{VMM}}$ by activating GABAergic neurons in the vlPAG during wakefulness, whereas GABAergic neurons in the $\mathrm{CeA}$ may disinhibit Glu ${ }^{\mathrm{SLD} \rightarrow \mathrm{VMM}}$ by suppressing vlPAG during REM and cataplexy.

As for another candidate region controlling $\mathrm{Glu}$ SLD $\rightarrow \mathrm{VMM}$ neurons, cholinergic neurons in the LDT have been suggested to activate Glu $^{\text {SLD }}$ neurons (Fuller et al., 2007; Peever and Fuller, 2016). Our cTRIO experiments revealed that input neurons in the SLD are not cholinergic but are apposed by cholinergic neurons in the LDT (Fig. 4B,C). Since cholinergic systems have long been assumed to be involved in the generation of REM sleep (George et al., 1964; Velazquez-Moctezuma et al., 1991; Van Dort et al., 2015), it is reasonable to presume that Glu ${ }^{\mathrm{SLD} \rightarrow \mathrm{VMM}}$ neurons might receive inputs from cholinergic neurons in the LDT.

We conclude that the Glut ${ }^{\mathrm{SLD}} \rightarrow \mathrm{Gly}^{\mathrm{VMM}} \rightarrow$ Mn neuronal pathway is mobilized as a common neural circuit during both REM sleep and cataplexy. In addition, we demonstrated that Gly ${ }^{\mathrm{VMM}} \rightarrow \mathrm{Mn}$ neurons innervate somatic motoneurons regulating all anti-gravity muscles except extraocular muscles, which is consistent with the defining feature of REM sleep. Our findings delineate the neural connectivity regulating muscle atonia, which exhibits shared neuronal machinery during REM sleep and cataplexy, and contribute to understanding the physiological role of REM sleep.

\section{References}

Boissard R, Gervasoni D, Schmidt MH, Barbagli B, Fort P, Luppi PH (2002) The rat ponto-medullary network responsible for paradoxical sleep onset and maintenance: a combined microinjection and functional neuroanatomical study. Eur J Neurosci 16:1959-1973.

Brooks PL, Peever JH (2011) Impaired GABA and glycine transmission triggers cardinal features of rapid eye movement sleep behavior disorder in mice. J Neurosci 31:7111-7121.

Capelli P, Pivetta C, Esposito MS, Arber S (2017) Locomotor speed control circuits in the caudal brainstem. Nature 551:373-377. 
Chase MH, Soja PJ, Morales FR (1989) Evidence that glycine mediates the postsynaptic potentials that inhibit lumbar motoneurons during the atonia of active sleep. J Neurosci 9:743-751.

Chemelli RM, Willie JT, Sinton CM, Elmquist JK, Scammell T, Lee C, Richardson JA, Williams SC, Xiong Y, Kisanuki Y, Fitch TE, Nakazato M, Hammer RE, Saper CB, Yanagisawa M (1999) Narcolepsy in orexin knockout mice: molecular genetics of sleep regulation. Cell 98:437-451.

Clément O, Sapin E, Bérod A, Fort P, Luppi PH (2011) Evidence that neurons of the sublaterodorsal tegmental nucleus triggering paradoxical (REM) sleep are glutamatergic. Sleep 34:419-423.

Erickson ETM, Ferrari LL, Gompf HS, Anaclet C (2019) Differential role of pontomedullary glutamatergic neuronal populations in sleep-wake control. Front Neurosci 13:1-16.

Eulenburg V, Armsen W, Betz H, Gomeza J (2005) Glycine transporters: essential regulators of neurotransmission. Trends Biochem Sci 30:325333.

Franklin KBJ, Paxinos GP (2001) The mouse brain in stereotaxic coordinates. Amsterdam: Elsevier.

Fuller PM, Saper CB, Lu J (2007) The pontine REM switch: past and present. J Physiol 584:735-741.

Garcia SV, Brischoux F, Clément O, Libourel P, Arthaud S, Lazarus M, Luppi P, Fort P (2018) Ventromedial medulla inhibitory neuron inactivation induces REM sleep without atonia and REM sleep behavior disorder. Nat Commun 9:504.

George R, Haslett WL, Jenden DJ (1964) A cholinergic mechanism in the brainstem reticular formation: induction of paradoxical sleep. Neuropharmacology 3:541-552.

Goulding M (2009) Circuits controlling vertebrate locomotion: moving in a new direction. Nat Rev Neurosci 10:507-518.

Gutierrez Herrera C, Girard F, Bilella A, Gent TC, Roccaro-Waldmeyer DM, Adamantidis A, Celio MR (2019) Neurons in the Nucleus papilio contribute to the control of eye movements during REM sleep. Nat Commun 10:5225.

Han W, Tellez LA, Rangel MJ, Motta SC, Zhang X, Perez IO, Canteras NS, Shammah-Lagnado SJ, van den Pol AN, de Araujo IE (2017) Integrated control of predatory hunting by the central nucleus of the amygdala. Cell 168:311-324.e18.

Hara J, Beuckmann CT, Nambu T, Willie JT, Chemelli RM, Sinton CM, Sugiyama F, Yagami KI, Goto K, Yanagisawa M, Sakurai T (2001) Genetic ablation of orexin neurons in mice results in narcolepsy, hypophagia, and obesity. Neuron 30:345-354.

Hasegawa E, Sakurai T, Mieda M, Hasegawa E, Yanagisawa M, Sakurai T, Mieda M (2014) Orexin neurons suppress narcolepsy via 2 distinct efferent pathways. J Clin Invest 124:604-616.

Hasegawa E, Maejima T, Yoshida T, Masseck OA, Herlitze S, Yoshioka M, Sakurai T, Mieda M (2017) Serotonin neurons in the dorsal raphe mediate the anticataplectic action of orexin neurons by reducing amygdala activity. Proc Natl Acad Sci USA 114:E3526-E3535.

Hirrlinger J, Marx G, Besser S, Sicker M, Köhler S, Hirrlinger PG, Wojcik SM, Eulenburg V, Winkler U, Hülsmann S (2019) GABA-glycine cotransmitting neurons in the ventrolateral medulla: development and functional relevance for breathing. Front Cell Neurosci 13:1-14.

Holstege JC, Bongers CMH (1991) A glycinergic projection from the ventromedial lower brainstem to spinal motoneurons. An ultrastructural double labelling study in rat. Brain Res 566:308-315.

Iranzo A, Santamaria J, Tolosa E (2016) Idiopathic rapid eye movement sleep behaviour disorder: diagnosis, management, and the need for neuroprotective interventions. Lancet Neurol 15:405-419.

Jouvet M (1962) Research on the neural structures and responsible mechanisms in different phases of physiological sleep. Arch Ital Biol 100:125-206.

Lai YY, Kodama T, Schenkel E, Siegel JM (2010) Behavioral response and transmitter release during atonia elicited by medial medullary stimulation. J Neurophysiol 104:2024-2033.

Lu J, Sherman D, Devor M, Saper CB (2006) A putative flip-flop switch for control of REM sleep. Nature 441:589-594.

Madisen L, Zwingman TA, Sunkin SM, Oh SW, Zariwala HA, Gu H, Ng LL, Palmiter RD, Hawrylycz MJ, Jones AR, Lein ES, Zeng H (2010) A robust and high-throughput Cre reporting and characterization system for the whole mouse brain. Nat Neurosci 13:133-140.

Mahoney CE, Agostinelli LJ, Brooks JNK, Lowell BB, Scammell TE (2017) GABAergic neurons of the central amygdala promote cataplexy. J Neurosci 37:3995-4006.
Morales FR, Sampogna S, Rampon C, Luppi PH, Chase MH (2006) Brainstem glycinergic neurons and their activation during active (rapid eye movement) sleep in the cat. Neuroscience 142:37-47.

Nabekura J, Katsurabayashi S, Kakazu Y, Shibata S, Matsubara A, Jinno S, Mizoguchi Y, Sasaki A, Ishibashi H (2004) Developmental switch from GABA to glycine release in single central synaptic terminals. Nat Neurosci 7:17-23.

Nishino S, Ripley B, Overeem S, Lammers GJ, Mignot E (2000) Hypocretin (orexin) deficiency in human narcolepsy. Lancet 355:39-40.

O’Brien JA, Berger AJ (1999) Cotransmission of GABA and glycine to brain stem motoneurons. J Neurophysiol 82:1638-1641.

Oishi Y, Williams RH, Agostinelli L, Arrigoni E, Fuller PM, Mochizuki T, Saper CB, Scammell TE (2013) Role of the medial prefrontal cortex in cataplexy. J Neurosci 33:9743-9751.

Oka T, Tsumori T, Yokota S, Yasui Y (2008) Neuroanatomical and neurochemical organization of projections from the central amygdaloid nucleus to the nucleus retroambiguus via the periaqueductal gray in the rat. Neurosci Res 62:286-298.

Osakada F, Callaway EM (2013) Design and generation of recombinant rabies virus vectors. Nat Protoc 8:1583-1601.

Peever J, Fuller PM (2016) Neuroscience: a distributed neural network controls REM sleep. Curr Biol 26:R34-R35.

Peever J, Luppi PH, Montplaisir J (2014) Breakdown in REM sleep circuitry underlies REM sleep behavior disorder. Trends Neurosci 37:279-288.

Peyron C, Tighe DK, Van Den Pol AN, De Lecea L, Heller HC, Sutcliffe JG, Kilduff TS (1998) Neurons containing hypocretin (orexin) project to multiple neuronal systems. J Neurosci 18:9996-10015.

Peyron C, Faraco J, Rogers W, Ripley B, Overeem S, Charnay Y, Nevsimalova S, Aldrich M, Reynolds D, Albin R, Li R, Hungs M, Pedrazzoli M, Padigaru M, Kucherlapati M, Fan J, Maki R, Lammers GJ, Bouras C, Kucherlapati R, et al. (2000) A mutation in a case of early onset narcolepsy and a generalized absence of hypocretin peptides in human narcoleptic brains. Nat Med 6:991-997.

Poyatos I, Ponce J, Aragón C, Giménez C, Zafra F (1997) The glycine transporter GLYT2 is a reliable marker for glycine-immunoreactive neurons. Brain Res Mol Brain Res 49:63-70.

Rizvi TA, Ennis M, Behbehani MM, Shipley MT (1991) Connections between the central nucleus of the amygdala and the midbrain periaqueductal gray: topography and reciprocity. J Comp Neurol 303:121-131.

Sakai K (2015) Paradoxical (rapid eye movement) sleep-on neurons in the laterodorsal pontine tegmentum in mice. Neuroscience 310:455-471.

Sakurai T (2007) The neural circuit of orexin (hypocretin): maintaining sleep and wakefulness. Nat Rev Neurosci 8:171-181.

Sakurai T (2014) The role of orexin in motivated behaviours. Nat Rev Neurosci 15:719-731.

Scammell TE (2015) Narcolepsy. N Engl J Med 373:2654-2662.

Scammell TE, Arrigoni E, Lipton JO (2017) Neural circuitry of wakefulness and sleep. Neuron 93:747-765.

Scammell TE, Willie JT, Guilleminault C, Siegel JM; International Working Group on Rodent Models of Narcolepsy (2009) A consensus definition of cataplexy in mouse models of narcolepsy. Sleep 32:111-116.

Schenck CH, Bundlie SR, Ettinger MG, Mahowald MW (1986) Chronic behavioral disorders of human REM sleep: a new category of parasomnia. Sleep 9:293-308.

Schiavo G, Benfenatit F, Poulain B, Rossetto O, Polverino de Laureto P, DasGupta BR, Montecucco C (1992) Tetanus and botulinum-B neurotoxins block neurotransmitter release by proteolytic cleavage of synaptobrevin. Nature 359:832-835.

Schwarz LA, Miyamichi K, Gao XJ, Beier KT, Weissbourd B, Deloach KE, Ren J, Ibanes S, Malenka RC, Kremer EJ, Luo L (2015) Viral-genetic tracing of the input-output organization of a central noradrenaline circuit. Nature 524:88-92.

Siegel JM, Nienhuis R, Fahringer HM, Paul R, Shiromani P, Dement WC, Mignot E, Chiu C (1991) Neuronal activity in narcolepsy: identification of cataplexy-related cells in the medial medulla. Science 252:1315-1318.

Soja PJ, López-Rodríguez F, Morales FR, Chase MH (1991) The postsynaptic inhibitory control of lumbar motoneurons during the atonia of active sleep: effect of strychnine on motoneuron properties. J Neurosci 11:2804-2811.

Stanek IE, Cheng S, Takatoh J, Han BX, Wang F (2014) Monosynaptic premotor circuit tracing reveals neural substrates for oro-motor coordination. Elife 3:e02511. 
Takeuchi T, Nomura T, Tsujita M, Suzuki M, Fuse T, Mori H, Mishina M (2002) Flp recombinase transgenic mice of C57BL/6 strain for conditional gene targeting. Biochem Biophys Res Commun 293:953-957.

Tanaka I, Ezure K (2004) Overall distribution of GLYT2 mRNA-containing versus GAD67 mRNA-containing neurons and colocalization of both mRNAs in midbrain, pons, and cerebellum in rats. Neurosci Res 49:165178.

Tanaka I, Ezure K, Kondo M (2003) Distribution of glycine transporter 2 mRNA-containing neurons in relation to glutamic acid decarboxylase mRNA-containing neurons in rat medulla. Neurosci Res 47:139-151.

Thannickal TC, Moore RY, Nienhuis R, Ramanathan L, Gulyani S, Aldrich M, Cornford M, Siegel JM (2000) Reduced number of hypocretin neurons in human narcolepsy. Neuron 27:469-474.

Torontali ZA, Fraigne JJ, Sanghera P, Horner R, Torontali ZA, Fraigne JJ, Sanghera P, Horner R, Peever J (2019) The sublaterodorsal tegmental nucleus functions to couple brain state and motor activity during REM sleep and wakefulness. Curr Biol 29:3803-3813.

Tovote P, Esposito MS, Botta P, Chaudun F, Fadok JP, Markovic M, Wolff SBE, Ramakrishnan C, Fenno L, Deisseroth K, Herry C, Arber S, Lüthi A (2016) Midbrain circuits for defensive behaviour. Nature 534:206-212.

Valencia Garcia S, Libourel P-A, Lazarus M, Grassi D, Luppi PH, Fort P (2017) Genetic inactivation of glutamate neurons in the rat sublaterodorsal tegmental nucleus recapitulates REM sleep behaviour disorder. Brain 140:414-428.

Van Dort CJ, Zachs DP, Kenny JD, Zheng S, Goldblum RR, Gelwan NA, Ramos DM, Nolan MA, Wang K, Weng FJ, Lin Y, Wilson MA, Brown
EN (2015) Optogenetic activation of cholinergic neurons in the PPT or LDT induces REM sleep. Proc Natl Acad Sci USA 112:584-589.

Velazquez-Moctezuma J, Shalauta M, Gillin JC, Shiromani PJ (1991) Cholinergic antagonists and REM sleep generation. Brain Res 543:175-179.

Vetrivelan R, Fuller PM, Tong Q, Lu J (2009) Medullary circuitry regulating rapid eye movement sleep and motor atonia. J Neurosci 29:9361-9369.

Vong L, Ye C, Yang Z, Choi B, Chua S, Lowell BB (2011) Leptin action on GABAergic neurons prevents obesity and reduces inhibitory tone to POMC neurons. Neuron 71:142-154.

Weber F, Chung S, Beier KT, Xu M, Luo L, Dan Y (2015) Control of REM sleep by ventral medulla GABAergic neurons. Nature 526:435-438.

Weber F, Hoang Do JP, Chung S, Beier KT, Bikov M, Saffari Doost M, Dan $Y$ (2018) Regulation of REM and non-REM sleep by periaqueductal GABAergic neurons. Nat Commun 9:354.

Weissbourd B, Ren J, DeLoach KE, Guenthner CJ, Miyamichi K, Luo L (2014) Presynaptic partners of dorsal raphe serotonergic and GABAergic neurons. Neuron 83:645-662.

Willie JT, Chemelli RM, Sinton CM, Tokita S, Williams SC, Kisanuki YY, Marcus JN, Lee C, Elmquist JK, Kohlmeier KA, Leonard CS, Richardson JA, Hammer RE, Yanagisawa M (2003) Distinct narcolepsy syndromes in orexin receptor-2 and orexin null mice: molecular genetic dissection of non-REM and REM sleep regulatory processes. Neuron 38:715-730.

Zhong P, Zhang Z, Barger Z, Ma C, Liu D, Ding X, Dan Y (2019) Control of non-REM sleep by midbrain neurotensinergic neurons. Neuron 104:795809.e6. 